\title{
Heat engines for dilatonic Born-Infeld black holes
}

\author{
Chandrasekhar Bhamidipati ${ }^{\mathrm{a}}$, Pavan Kumar Yerra ${ }^{\mathrm{b}}$ \\ School of Basic Sciences, Indian Institute of Technology Bhubaneswar, Bhubaneswar 751013, India
}

Received: 8 February 2017 / Accepted: 16 July 2017 / Published online: 9 August 2017

(C) The Author(s) 2017. This article is an open access publication

\begin{abstract}
In the context of dilaton coupled Einstein gravity with a negative cosmological constant and a Born-Infeld field, we study heat engines where a charged black hole is the working substance. Using the existence of a notion of thermodynamic mass and volume (which depend on the dilaton coupling), the mechanical work takes place via the $p d V$ terms present in the first law of extended gravitational thermodynamics. The efficiency is analyzed as a function of dilaton and Born-Infeld couplings, and the results are compared with analogous computations in the related conformal solutions in the Brans-Dicke-Born-Infeld theory and black holes in anti-de Sitter space-time.
\end{abstract}

\section{Introduction}

Recent interest in treating the cosmological constant $\Lambda$ as a dynamical parameter [1-15] has led to important extensions of the classical thermodynamic properties of a black hole [16-19], which relates the mass $M$, surface gravity $\kappa$, and outer horizon area $A$ of a black hole solution to the energy, temperature, and entropy ( $U, T$, and $S$, resp.) according to (in geometrical units where $G, c, \hbar, k_{\mathrm{B}}$ are set to unity)

$M=U, \quad T=\frac{\kappa}{2 \pi}, \quad S=\frac{A}{4}$.

Now, the cosmological constant treated as pressure $p=$ $-\Lambda / 8 \pi$, has a conjugate variable, the thermodynamic volume $V$ associated with the black hole. In this extended thermodynamics, temperature and entropy continue to be related to surface gravity and area as usual, while the mass, however, turns out to be related to enthalpy $H$ [5]: $M=H \equiv U+p V$. The first law now reads

$\mathrm{d} M=T \mathrm{~d} S+V \mathrm{~d} p$

\footnotetext{
${ }^{a}$ e-mail: chandrasekhar@iitbbs.ac.in

b e-mail: pk11@iitbbs.ac.in
}

The black holes may have other parameters such as gauge charges, angular momentum, coupling constants (GaussBonnet, Born-Infeld) which enter additively with their conjugates in the first law (1.2) in the usual way. For static black holes, the thermodynamic volume $V$ is just the geometric volume (defined in terms of the horizon radius $r_{+}$) of the black hole in question [20], but, in general, the two volumes differ, leading to novel physics such as in rotating black holes, AdS-Taub-nut geometry, and black holes with dilaton fields (see for instance [7,21-26]). An extended thermodynamical phase space treatment leads to an exact identification of small to large black hole phase transition in charged AdS and related black holes to a van der Waals liquid-gas phase transition $[27,28]$, including an exact map of critical exponents. Furthermore, the phase transitions occur in the $p-T$ plane as opposed to the $Q-T$ plane and hence identical parameters are now being compared on both sides [29].

The possibility of extracting mechanical work from heat energy via the $p d V$ term present in Eq. (1.2) has led to the proposal of a holographic heat engine in [30], where the working substance is a black hole solution of the gravity system. Several holographic engines have since been studied [31-34]. The black hole in particular provides an equation of state. Work can be extracted from such an engine by defining a cycle in state space where there is a net input heat flow $Q_{H}$, a net output heat flow $Q_{C}$, and a net output work $\mathrm{W}$, such that $Q_{H}=W+Q_{C}$.

The efficiency of such heat engines can be written in the usual way as $\eta=W / Q_{H}=1-Q_{C} / Q_{H}$. Its value depends crucially on the equation of state provided by the black hole and the choice of the cycle in state space. Considering the thermodynamical cycle in Fig. 1 advocated in $[30,35,36]$ for static black holes, the entropy and the volume turn out to be dependent on each through $r_{+}$. This means that isochores are adiabats, and hence the heat flows in the cycle in Fig. 1 occur only along top and bottom lines. Formal computation of the efficiency proceeds via the evaluation of $\int C_{p} d T$ along those isobars, where $C_{p}$ is the specific heat at constant pressure. 

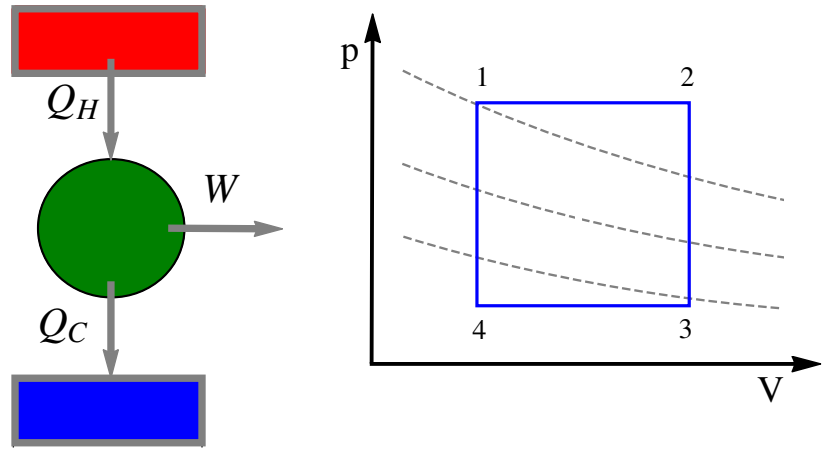

Fig. 1 Heat engine and a rectangular cycle with flows

This in general being difficult, efficiency was evaluated in various limits in $[35,36]$. An exact formula for efficiency,

$\eta=1-\frac{M_{3}-M_{4}}{M_{2}-M_{1}}$

was later obtained in [37], using the fact that the mass of the black hole is just the enthalpy and total heat flow along an isobar is the change in enthalpy. For static black holes, $M$ can be written as a function of $p$ and $V$.

Let us note that defining heat engines via cycles in state space (with dynamical cosmological constant) represents a journey through a family of holographically dual field theories [38-42] (at large $N_{c}$ ). The exact holographic dictionary corresponding to heat engines and their efficiency needs further study. Nevertheless, we restrict to applications to black holes in Einstein gravity with higher derivative corrections, which are interesting on their own right. Einstein gravity is considered to be an effective description of the underlying quantum gravity, such as string theory in the low energy limit. Thus, it is interesting to study the effect of stringy corrections on heat engines and their efficiency. The effect of Gauss-Bonnet and Born-Infeld higher curvature corrections on efficiency was analyzed in $[35,36]$.

In this paper, we study the efficiency of heat engines in the presence of another stringy effect, namely, the dilaton field (Einstein gravity non-minimally coupled to a dilaton is present in the low energy limit of string theory [43]). In particular, we consider the Einstein-Maxwell dilaton system in the presence of two Liouville type potentials and also dilatonic black holes coupled to nonlinear Born-Infeld theory (Dilatonic-BI) in an extended phase space, in fixed charge ensemble [44]. We also consider its conformally consistent counter part, the Brans-Dicke Born-Infeld theory (BD-BI) studied in [45]. BD (Brans-Dicke) theory has been significant in the explanation of the cosmic inflation [46], and consistent with Dirac's large number hypothesis and Mach's principle $[47,48]$. Thermodynamics of charged black holes in Brans-Dicke theory have been studied in [49-52]. This theory produces the solar system experimental observations with a specific domain of BD parameter $\omega$ [53].

Moreover, the presence of the dilaton field in EinsteinMaxwell theory changes the causal structure of the spacetime and modifies the thermodynamic properties of the black holes in a non-trivial way. Rich structure and $p$ $V$ criticality in black holes with higher derivative couplings, Born-Infeld and dilaton black holes have been studied earlier. ${ }^{1}$ In the case, when there are Liouville type potentials for the scalar fields, the solution is asymptotically neither flat nor AdS. Furthermore, an exponential or Liouville potential represents the higher-dimensional cosmological constant present in non-critical string theories [74-79], non-trivial curved adS backgrounds [80], or leading $g_{s}$ corrections to critical string theories in a flat background. Starting from standard AdS/CFT duality in higher dimensions, holography of models with Liouville type scalar potentials are generated upon dimensional reduction procedure [81]. In [82], it has been conjectured that the linear dilaton space-times, which arise as nearhorizon limits of dilatonic black holes, might exhibit holography.

Non-asymptotically flat/AdS black hole space-times have been actively studied for possible extensions of holography [82-96]. The usual notions of thermodynamic mass, thought of as enthalpy of a space-time, akin to an AdS black hole go through for more general backgrounds. For instance, in the context of black holes in Liftshitz space-times (which are asymptotically neither AdS nor flat) [97,98], it has been argued that introducing pressure ${ }^{2}$ together with thermodynamic volume and studying extended thermodynamic phase structure (in spite of the fact that all thermodynamic quantities now depend on the dilaton coupling constant) is physically and holographically sensible, with applications to condensed matter systems and quantum criticality. A holographic interpretation for the Van der Waals transition was proposed in [30], within the extended phase thermodynamics, where varying the cosmological constant in the bulk corresponds to perturbing the dual CFT, triggering a renormalization group flow. The transition is then interpreted not as a thermodynamical transition but, instead, as a transition in the space of field theories. Having scalar fields in the bulk (such as the charged Dilaton system in the present manuscript and other examples [33,97]) might turn on certain operators in the boundary theory triggering a non-trivial RG flow. In particular, there might be solutions of dilatonic theories with Liouville type poten-

\footnotetext{
${ }^{1}$ See for instance [23-26,54-73].

${ }^{2}$ Recently, there have also been much more general proposals, that pressure should be introduced not just for black holes, but for all spacetimes, resulting in a generalized notion of thermodynamic volume [21].
} 
tials connecting the IR dynamics to AdS asymptotics in UV [99].

Also, pressure for asymptotically non-flat/ads black holes with Liouville type potentials has been introduced and the corresponding PV criticality studied in good detail in [68] and extended to conformally coupled scalars, i.e., the BransDicke-Born-Infeld solutions [45]. Following these works, and the existence of an extended first law with pressure and volume, including the presence of PV criticality allows us to naturally define a heat engine, exactly as in the examples considered for AdS, leading to extension of the results of $[30,44,45,68]$. The working substance is still the charged black hole, however, the efficiency of heat engines will now depend on the coupling constants provided by dilaton and Born-Infeld theories. A feature of our heat engines is that the volume depends on the coupling constant of dilaton $(\alpha)$ and electromagnetic fields $(\beta)$ and is not same as the geometrical volume. Existence of an exact formula allows us to study efficiency as a function of both couplings, i.e., $\eta=\eta(\alpha, \beta)$ and take various limits where we keep $\alpha$ fixed while tuning $\beta$ and vice versa. In particular, in the limit $\alpha \rightarrow 0$ and in the high temperature limit, our exact results agree with the effect of Born-Infeld field on efficiency, captured in [36].

\section{Heat engines from charged black holes in Dilatonic and Brans-Dicke-Born-Infeld theories}

Following the discussion of last section, where a cycle in state space was presented for heat engines from charged black holes, we continue with the computation of the efficiency of such engines. We first study the dilatonic Born-Infeld model and later study the corrections to efficiency of heat engines in Brans-Dicke-Born-Infeld model.

\subsection{Dilatonic Born-Infeld model}

For the purpose of computing efficiency, we start from the relevant expression for mass of the Born-Infeld dilaton black hole [44] (details of black hole solutions are reproduced in "Appendix A" for reference),

$M=\frac{b^{(n-1) \gamma}(n-1) \omega_{n-1}}{16 \pi\left(\alpha^{2}+1\right)} m$,

where $b$ is an arbitrary positive constant, $\alpha$ is the dilaton coupling constant, $n$ represents the number of spatial dimensions (we restrict to $n>3$ ) and $\omega_{n-1}$ is the volume of the constant curvature hypersurface characterizing the horizon . Using the expression for $m$ (see "Appendix A"), the mass can be expressed in terms of other thermodynamic parameters as

$$
\begin{aligned}
M\left(r_{+}, p\right)= & \frac{b^{(n-1) \gamma}\left(\alpha^{2}+1\right) \omega_{n-1}}{16 \pi} r_{+}^{n(1-\gamma)-\gamma} \\
& \times\left\{\frac{k(n-1)(n-2) b^{-2 \gamma}}{\left(1-\alpha^{2}\right)\left(n+\alpha^{2}-2\right)} r_{+}^{4 \gamma-2}+\frac{16 \pi p}{\left(n+\alpha^{2}\right)} r_{+}^{2 \gamma}\right. \\
& -\frac{4 \beta^{2} b^{2 \gamma}}{\left(\alpha^{2}-n\right)} \times\left(1-2 F_{1}\left(\left[-\frac{1}{2}, \frac{\alpha^{2}-n}{2 n-2}\right]\right.\right. \\
& {\left.\left.\left.\left[\frac{\alpha^{2}+n-2}{2 n-2}\right],-\eta_{+}\right)\right)\right\} . }
\end{aligned}
$$

A few comments are in order. Here, $p$ is the pressure and $\beta$ is the Born-Infeld parameter, where $\beta \rightarrow \infty$ corresponds to the Maxwell limit. $\gamma=\alpha^{2} /\left(\alpha^{2}+1\right)$ and $\eta_{+}=\eta\left(r=r_{+}\right)$with $\eta=\frac{q^{2} b^{2 \gamma(1-n)}}{\beta^{2} r^{2(n-1)(1-\gamma)}}$ and $r_{+}$is the radius of the horizon. $k(>0)$ is constant characterizing the $(n-1)$ dimensional hypersurface. The temperature expressed as a function of other thermodynamic parameters is

$$
\begin{aligned}
T= & \frac{\left(\alpha^{2}+1\right)}{4 \pi(n-1)}\left(\frac{k(n-2)(n-1) b^{-2 \gamma}}{\left(1-\alpha^{2}\right)} r_{+}^{2 \gamma-1}\right. \\
& \left.+16 \pi p \frac{\left(n-\alpha^{2}\right)}{\left(n+\alpha^{2}\right)} r_{+}+\frac{4 \beta^{2} b^{2 \gamma}}{r_{+}^{2 \gamma-1}}\left(1-\sqrt{1+\eta_{+}}\right)\right) .
\end{aligned}
$$

The thermodynamic volume $V$ is different from the geometrical volume due to the dependence on $\gamma$ [44],

$V=\frac{b^{(n-1) \gamma} r_{+}^{n-\gamma(n-1)}}{n-\gamma(n-1)} \omega_{n-1}$.

Now the equation of state $p(V, T)$ for our working substance in the $p-r_{+}$plane, or equivalently the $p-V$ plane (using Eq. (2.4)) is [44],

$$
\begin{aligned}
p= & \frac{\Gamma T}{r_{+}}-\frac{k(n-2)\left(1+\alpha^{2}\right) \Gamma}{4 \pi\left(1-\alpha^{2}\right) b^{2 \gamma} r_{+}^{2-2 \gamma}}+\frac{\beta^{2}\left(n+\alpha^{2}\right) b^{2 \gamma}}{4 \pi\left(n-\alpha^{2}\right) r_{+}^{2 \gamma}} \\
& \times\left(\sqrt{1+\eta_{+}}-1\right)
\end{aligned}
$$

where $\Gamma=\frac{(n-1)\left(n+\alpha^{2}\right)}{4\left(n-\alpha^{2}\right)\left(\alpha^{2}+1\right)}$. A possible scheme for our heat engine (based on the cycle ${ }^{3}$ given in Fig. 1) involves specifying values of temperatures $\left(T_{2}, T_{4}\right)$ (which in turn fixes $\left.\left(T_{H}, T_{C}\right)\right)$ and volumes $\left(V_{2}, V_{4}\right)$. The pressures $p_{1}=p_{2}$ and $p_{4}=p_{3}$ have to be computed from the equation of state and depend on the couplings $\alpha$ and $\beta$. Since the radii $r_{1}, r_{3}$ can be obtained analogously and the mass $M$, written as a function of $r_{+}$and $p$ is as in Eq. (2.2), the efficiency of the engine can now be studied as a function of couplings $\alpha$ and $\beta$. Considering the cycle given in Fig. 1, the efficiency of heat engines can be defined entirely in terms of the black hole mass evaluated at the corners as given in (1.3). Notice that

\footnotetext{
$\overline{3 \text { (See Ref. [30] }}$, for reasons for this choice for static black holes).
} 
in the present scheme the Carnot efficiency $\eta_{\mathrm{C}}=1-\frac{T_{C}}{T_{H}}$, the upper bound to our engine efficiency working between highest and lowest temperatures $T_{H}$ and $T_{C}$, respectively, is fixed for all $\alpha, \beta$. Another useful quantity to compare with is the efficiency in the Einstein-Maxwell case $\eta_{0}{ }^{4}$

We first check the case where the dilaton coupling $\alpha$ is set to zero, in which case we have a pure Born-Infeld black hole. The efficiency of the heat engine in this case was studied in [36], in the high temperature limit. For $n=4$, efficiency (Eq. (1.3)) takes the form
In fact, for $n=3$, for large volume branch of solutions and neglecting $q$ to leading order, we have

$$
\begin{aligned}
\left.\eta\right|_{(\alpha=0, \beta \rightarrow \infty)}= & \left(1-\frac{p_{4}}{p_{1}}\right)\left\{1-\frac{3}{8 p_{1}}\left(\frac{S_{2}^{1 / 2}-S_{1}^{1 / 2}}{S_{2}^{3 / 2}-S_{1}^{3 / 2}}\right)\right. \\
& \left.+O\left(\frac{1}{p_{1}^{2}}\right)\right\} .
\end{aligned}
$$

This matches with equation (20) in [30].

From Fig. 2, it can be seen that both efficiency ratios, i.e., $\eta / \eta_{C}$ and $\eta / \eta_{0}$, grow slowly for a while and then rise,

$\left.\eta\right|_{\alpha=0}=\frac{\left(1-\frac{p_{4}}{p_{1}}\right)}{\left\{1+\frac{3 \sqrt{2}}{8 p_{1}\left(\sqrt{V_{3}}+\sqrt{V_{4}}\right)}+\frac{\beta^{2}}{4 \pi p_{1}}\left[1-\frac{\left({ }_{2}\left[-\frac{2}{3},-\frac{1}{2}, \frac{1}{3}, \frac{-\pi^{3} q^{2}}{2 \sqrt{2} \beta^{2} V_{3}^{3 / 2}}\right] V_{3}-2 F_{1}\left[-\frac{2}{3},-\frac{1}{2}, \frac{1}{3}, \frac{-\pi^{3} q^{2}}{2 \sqrt{2} \beta^{2} V_{4}^{3 / 2}}\right] V_{4}\right)}{\left(V_{3}-V_{4}\right)}\right]\right\}}$

when $\beta \rightarrow \infty$, this becomes

$\left.\eta\right|_{(\alpha=0, \beta \rightarrow \infty)}=\frac{\left(1-\frac{p_{4}}{p_{1}}\right)}{\left\{1+\frac{3 \sqrt{2}}{8 p_{1}\left(\sqrt{V_{3}}+\sqrt{V_{4}}\right)}-\frac{\sqrt{2} \pi^{2} q^{2}}{16 p_{1} \sqrt{V_{4}}\left(V_{3}+\sqrt{V_{3} V_{4}}\right)}\right\}}$

For large $p_{1}$, one obtains

$$
\begin{aligned}
\left.\eta\right|_{\alpha=0}= & \left(1-\frac{p_{4}}{p_{1}}\right) \\
& \times\left\{1-\frac{1}{p_{1}}\left(\frac{3 \sqrt{2}}{8\left(\sqrt{V_{3}}+\sqrt{V_{4}}\right)}+\frac{\beta^{2}}{4 \pi}\left[1-\frac{\left({ }_{2} F_{1}\left[-\frac{2}{3},-\frac{1}{2}, \frac{1}{3}, \frac{-\pi^{3} q^{2}}{2 \sqrt{2} \beta^{2} V_{3}^{3 / 2}}\right] V_{3}-{ }_{2} F_{1}\left[-\frac{2}{3},-\frac{1}{2}, \frac{1}{3}, \frac{-\pi^{3} q^{2}}{2 \sqrt{2} \beta^{2} V_{4}^{3 / 2}}\right] V_{4}\right)}{\left(V_{3}-V_{4}\right)}\right]\right)\right. \\
& \left.+O\left(\frac{1}{p_{1}^{2}}\right)\right\}
\end{aligned}
$$

and

$$
\begin{aligned}
\left.\eta\right|_{(\alpha=0, \beta \rightarrow \infty)}= & \left(1-\frac{p_{4}}{p_{1}}\right)\left\{1-\frac{1}{p_{1}}\left(\frac{3 \sqrt{2}}{8\left(\sqrt{V_{3}}+\sqrt{V_{4}}\right)}\right.\right. \\
& \left.\left.-\frac{\sqrt{2} \pi^{2} q^{2}}{16 \sqrt{V_{4}}\left(V_{3}+\sqrt{V_{3} V_{4}}\right)}\right)+O\left(\frac{1}{p_{1}^{2}}\right)\right\} .
\end{aligned}
$$

in agreement with the results in [36] for high temperatures. We see from Fig. 3 that, an increase in $q$ causes significant changes in the efficiency. In fact, we can see the effect of various parameters ${ }^{5}$ on efficiency from Fig. 4.

We now keep $\alpha$ non-zero and study the resulting efficiency in the limit $\beta \rightarrow \infty$. Now $\eta$ (Eq. (1.3)) for $n=4$, can be expressed as

$$
\left.\eta\right|_{\beta \rightarrow \infty}=\frac{\left(1-\frac{p_{4}}{p_{1}}\right)}{\left\{1+\frac{\left(4+\alpha^{2}\right)}{8 p_{1} \pi\left(\alpha^{2}+2\right)\left(1-\alpha^{2}\right)}\left[\frac{V_{3}^{\left(\frac{2-\gamma}{4-3 \gamma}\right)}-V_{4}^{\left(\frac{2-\gamma}{4-3 \gamma}\right)}}{V_{3}-V_{4}}\right]\left(\frac{3 A_{o}^{2 \gamma-2}}{b^{2 \gamma}}+\frac{q^{2} A_{o}^{4 \gamma-6}\left(\alpha^{2}-1\right)}{b^{4 \gamma}\left(V_{3} V_{4}\right)^{\left(\frac{2-\gamma}{4-3 \gamma}\right)}}\right)\right\}}
$$

\footnotetext{
$\overline{4}$ (i.e., the limit $\alpha \rightarrow 0$ and $\beta \rightarrow \infty$, and we also rescaled the charge $q \rightarrow \sqrt{\frac{(n-1)(n-2)}{2}} q$ to get an exact Reissner-Nordstrom-anti-de Sitter black hole).
}

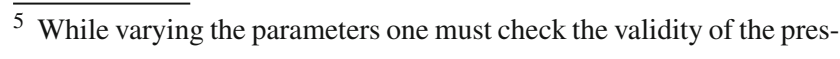
sures. 


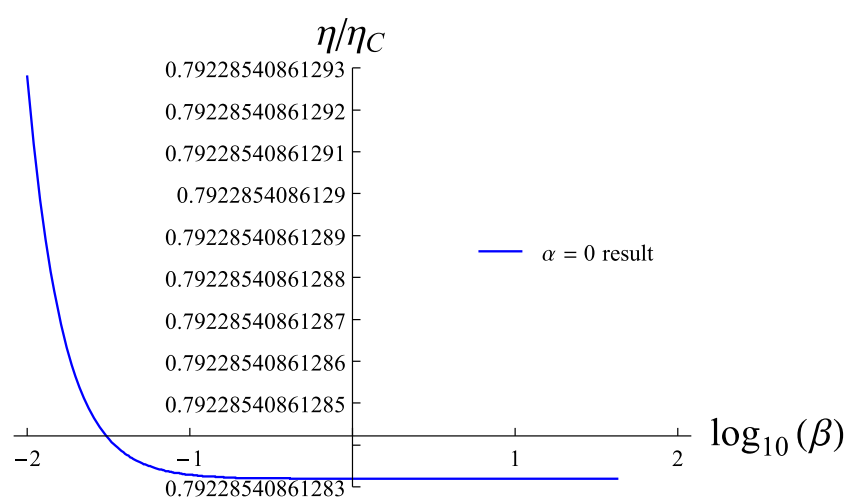

(a)

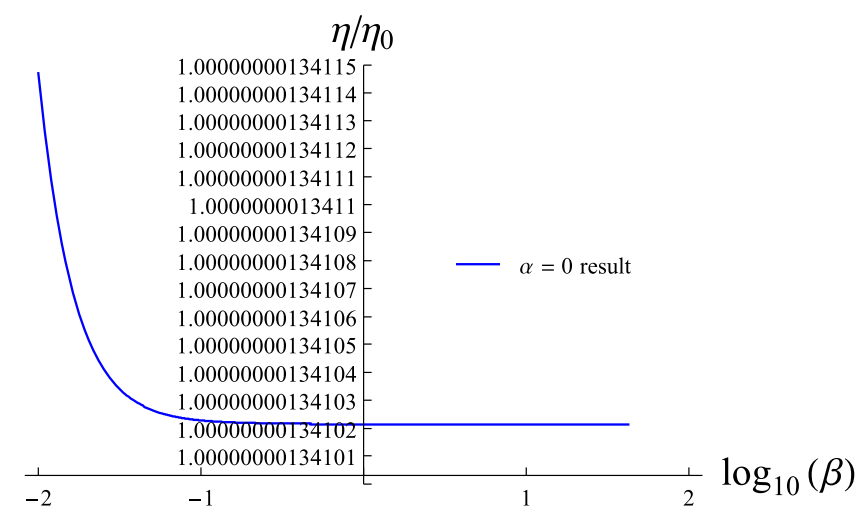

(b)

Fig. 2 For the case $\alpha=0$. a The ratio $\eta / \eta_{C}$ vs. $\log _{10}(\beta)$. b The ratio $\eta / \eta_{0}$ vs $\log _{10}(\beta)$. (Here, we have chosen the values $n=4, q=0.1, b=1$, $T_{4} \equiv T_{C}=30, T_{2} \equiv T_{H}=60, V_{2}=33000$, and $V_{4}=15500$ )

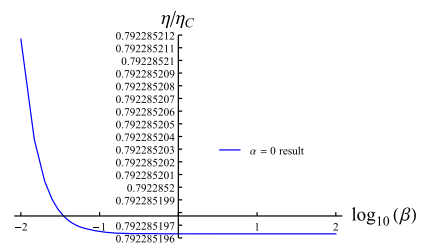

(a)

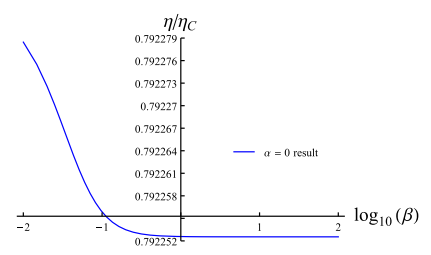

(b)

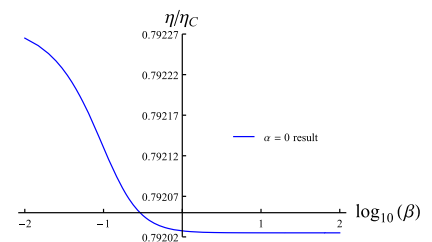

(c)

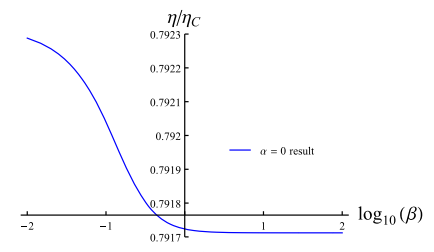

(d)

Fig. 3 Effect of q on efficiency, $\mathbf{a}$ for $q=2$, $\mathbf{b}$ for $q=25$, $\mathbf{c}$ for $q=70$ and $\mathbf{d}$ for $q=100$, when other parameters (see the caption of Fig. 2 for parameter values) are fixed

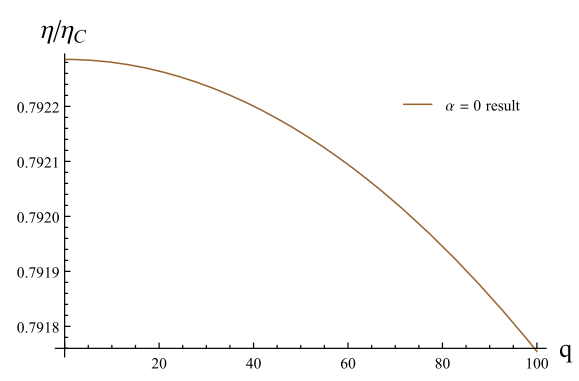

(a)

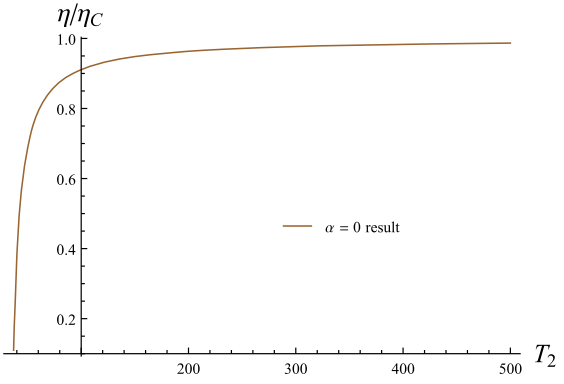

(b)

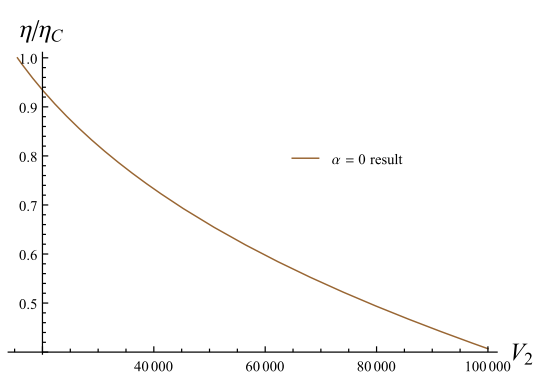

(c)

Fig. 4 Effect of parameters on efficiency, when other parameters (see the caption of Fig. 2 for parameter values) are fixed and $\beta=5$

For large $p_{1}$, one obtains

$$
\begin{aligned}
\left.\eta\right|_{\beta \rightarrow \infty}= & \left(1-\frac{p_{4}}{p_{1}}\right)\left\{1-\frac{\left(4+\alpha^{2}\right)}{8 p_{1} \pi\left(\alpha^{2}+2\right)\left(1-\alpha^{2}\right)}\right. \\
& \times\left[\frac{\left.V_{3}^{\left(\frac{2-\gamma}{4-3 \gamma}\right)}-V_{4}^{\left(\frac{2-\gamma}{4-3 \gamma}\right)}\right]}{V_{3}-V_{4}}\right] \\
& \times\left(\frac{3 A_{o}^{2 \gamma-2}}{b^{2 \gamma}}+\frac{q^{2} A_{o}^{4 \gamma-6}\left(\alpha^{2}-1\right)}{b^{4 \gamma}\left(V_{3} V_{4}\right)^{\left(\frac{2-\gamma}{4-3 \gamma}\right)}}\right) \\
& \left.+O\left(\frac{1}{p_{1}^{2}}\right)\right\}
\end{aligned}
$$

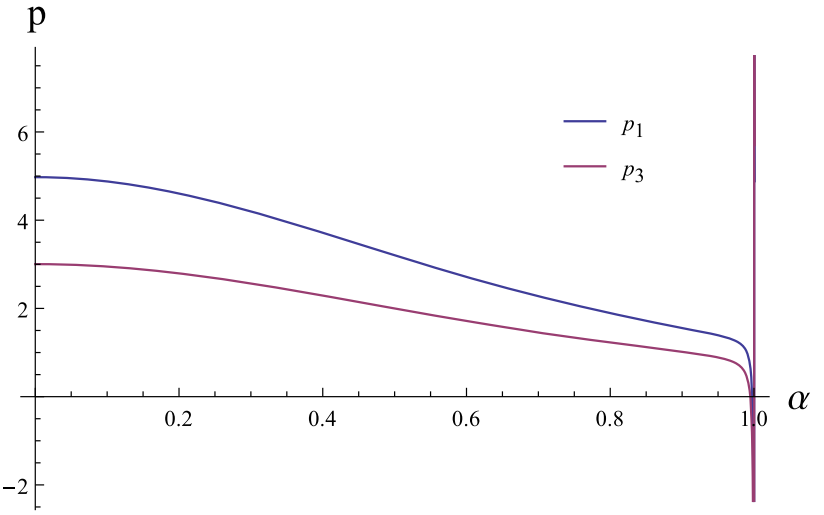

Fig. 5 In the limit $\beta \rightarrow \infty$, physical range of pressures (see the caption of Fig. 2 for parameter values) 


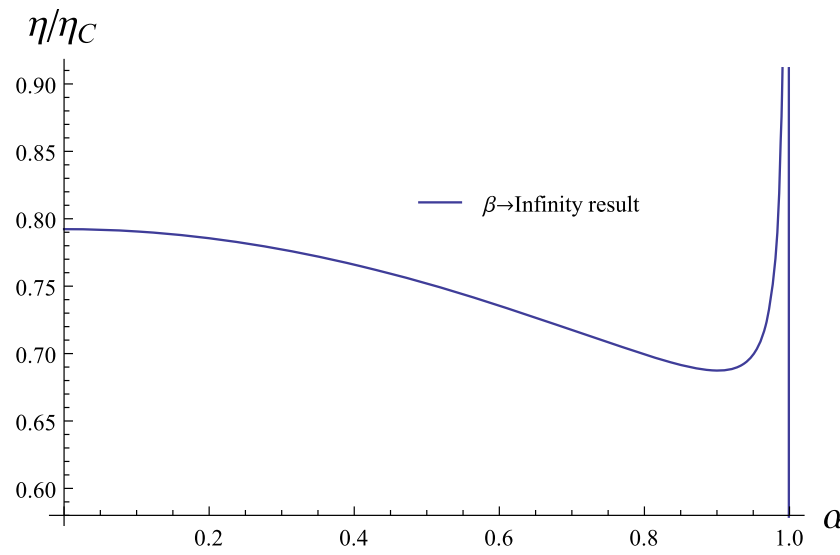

(a)

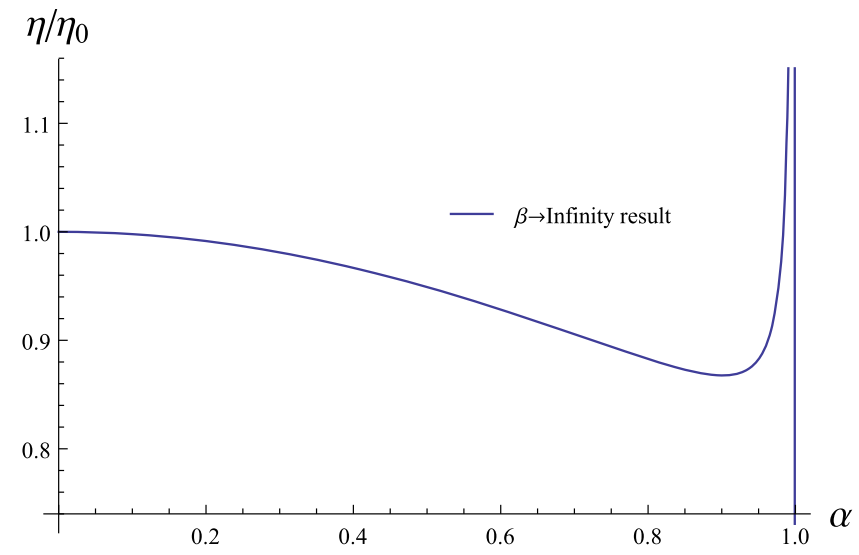

(b)

Fig. 6 In the limit $\beta \rightarrow \infty$. a The ratio $\eta / \eta_{C}$ vs. $\alpha$. b The ratio $\eta / \eta_{0}$ vs $\alpha$ (see the caption of Fig. 2 for parameter values)

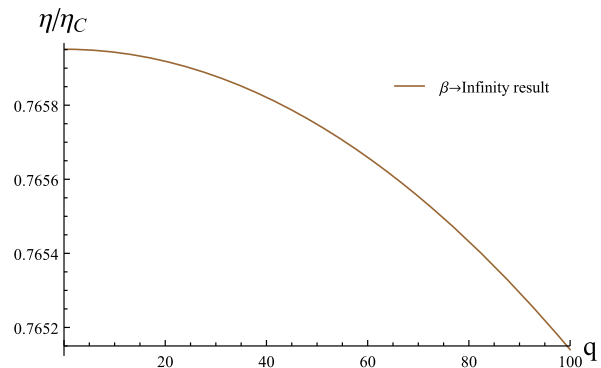

(a)

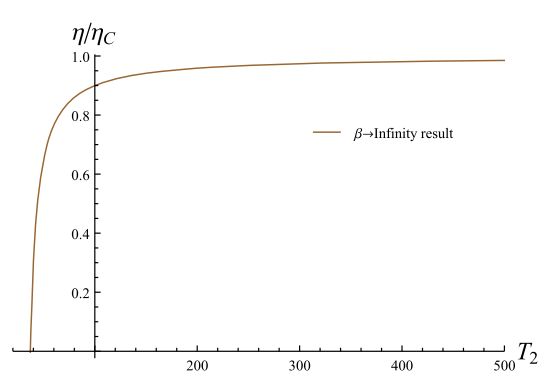

(b)

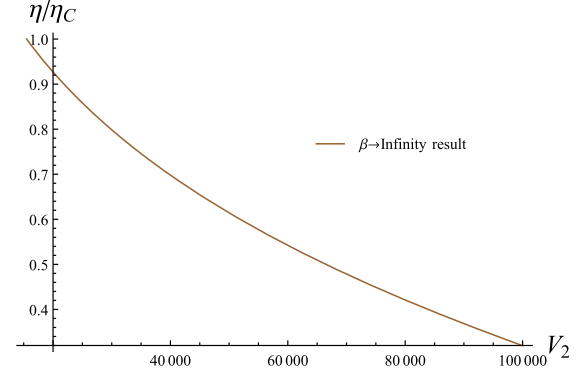

(c)

Fig. 7 Effect of parameters on efficiency, when other parameters (see the caption of Fig. 2 for parameter values) are fixed and $\alpha=0.4$

which shows the leading behavior of $\eta$ is $\left(1-p_{4} / p_{1}\right)$, where $A_{o}=\left(\frac{4-3 \gamma}{2 \pi^{2} b^{3 \gamma}}\right)^{1 /(4-3 \gamma)}$.

The efficiency of our engine now depends only on the dilatonic coupling and a comparison with both $\eta_{\mathrm{C}}$ and $\eta_{0}$ is again possible. Using the equation of state one can check whether the pressures $\left(p_{1}, p_{3}\right)$ in the engine remain physical as $\alpha$ changes. Since we have fixed $\left(T_{2}, V_{2}\right)$ and $\left(T_{4}, V_{4}\right)$, the pressures are now $\alpha$-dependent. In fact, the pressures become negative as $\alpha$ increases beyond 2 , diverging at $\alpha=1,2$ since the black hole solution is diverging at these points. If we consider the critical behavior of our black hole [44], the universal ratio $\rho_{c}$ is positive, provided $\alpha<1$, so we restrict ourselves to the physical range of $\alpha$, i.e., $0<\alpha<1$ (see Fig. 5).

A study of how the efficiency $\eta$ varies with respect to $\alpha$ shows that, initially, it falls as compared to $\eta_{\mathrm{C}}$, but then rises again (see Fig. 6). One notes that as $\alpha \rightarrow 1$, results on the efficiency are less reliable as pressure may no more be positive. Figure 7 shows a similar effect of various parameters on efficiency of the Born-Infeld case ${ }^{6}$

$\overline{6 \text { The plots for }} \frac{\eta}{\eta_{c}}$ vs $\alpha$ for different $q(=2,25,70,100)$ are similar to Fig. 6.
When we consider the effect of both couplings $(\alpha, \beta)$ on the efficiency of our engine (see Fig. 8), for a sample range of parameters $10^{-2}<\beta<10^{2}$ and $0<\alpha<1$ (we checked that the pressures are physical over this sample range of parameters), both ratios $\eta / \eta_{\mathrm{C}}$ and $\eta / \eta_{0}$ decrease rapidly in the turnaround region where, roughly, $10^{-2}<\beta<10^{-1}$ and become steady as $\beta$ increases, while as $\alpha$ increases, initially both the ratios decrease up to $\alpha \approx 0.91$ and then raise again.

\subsection{Brans-Dicke-Born-Infeld model}

Computation of corrections to efficiency in the Brans-Dicke Born-Infeld theory proceeds by writing down the relevant expression for enthalpy and equation of state [45] (details of black holes and thermodynamic quantities are summarized in "Appendix B") as

$$
\begin{aligned}
M\left(r_{+}, p\right)= & \frac{\varpi_{n-1} b^{(n-1) \gamma}\left(1+\alpha^{2}\right)(n-1)}{16 \pi} r_{+}^{(n-1)(1-\gamma)-1} \\
& \times\left\{\frac{(n-2) b^{-2 \gamma}}{\left(1-\alpha^{2}\right)\left(n+\alpha^{2}-2\right)} r_{+}^{2 \gamma}\right.
\end{aligned}
$$




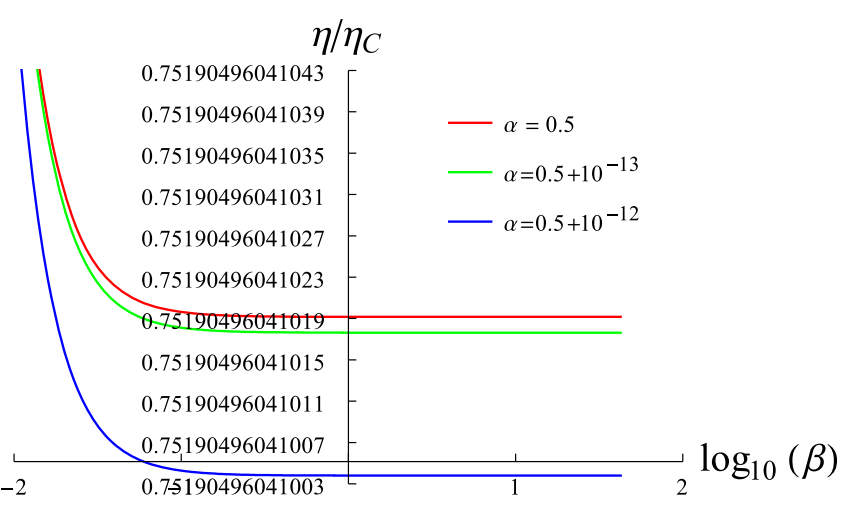

(a)

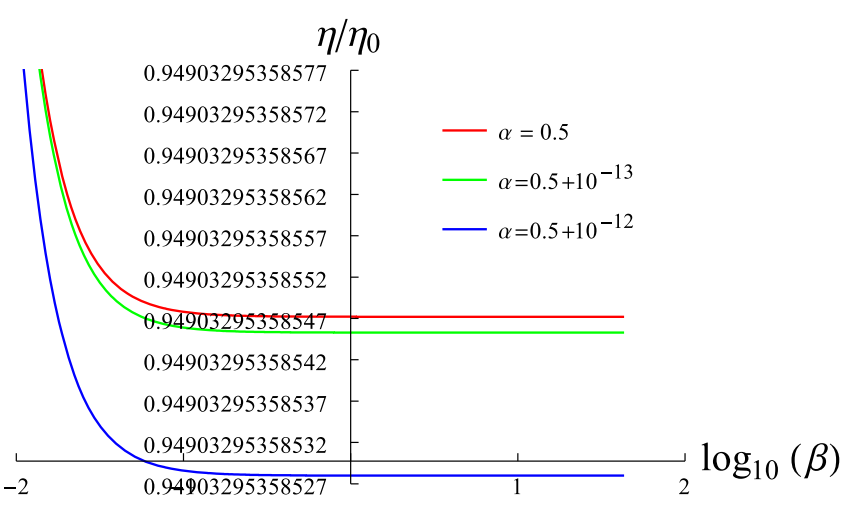

(b)

Fig. 8 For the case $\alpha \neq 0$ and $\beta \nrightarrow \infty$. a The ratio $\eta / \eta_{C}$ vs $\log _{10}(\beta)$. b The ratio $\eta / \eta_{0} \operatorname{vs} \log _{10}(\beta)$ (see the caption of Fig. 2 for parameter values)

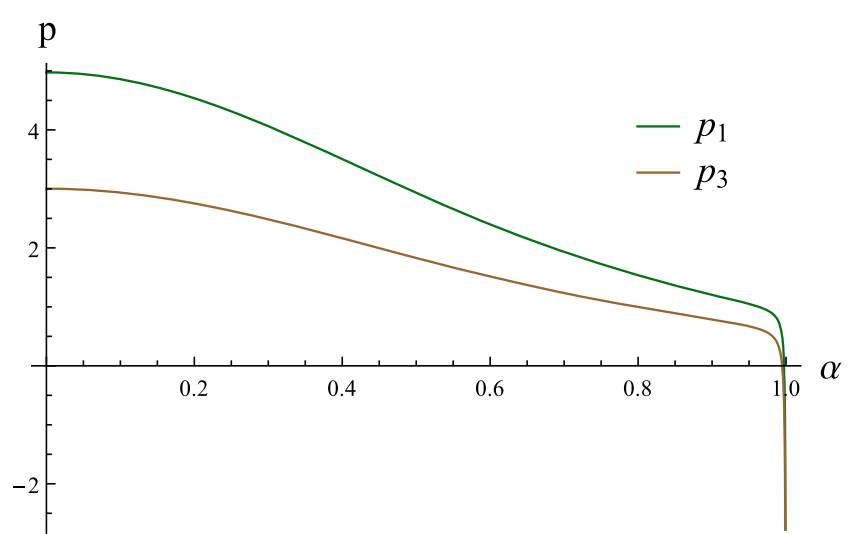

(a)

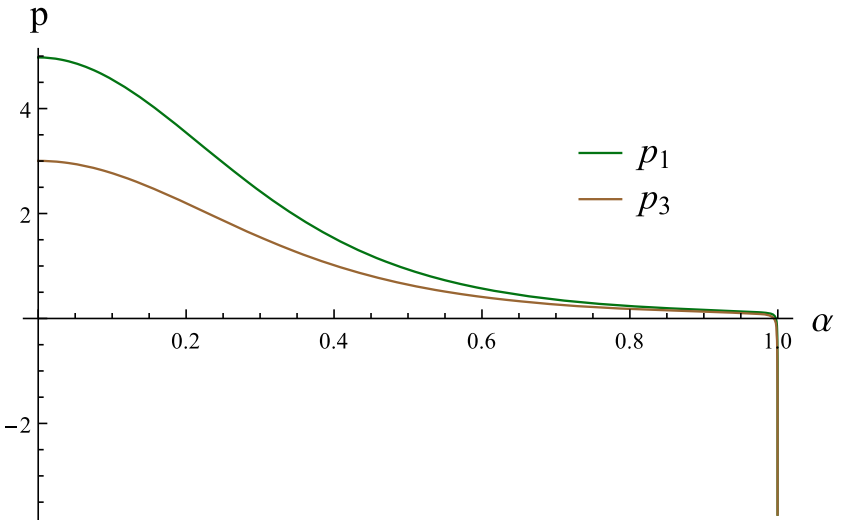

(b)

Fig. 9 The behavior of the pressures of upper isobar $\left(p_{1}\right)$ and lower isobar $\left(p_{3}\right)$ for engine cycle with respect to $\alpha$ in: a Einstein-BI-dilaton theory and $\mathbf{b}$ BD-BI theory. (see the caption of Fig. 2 for the parameter values)

$$
\begin{gathered}
-\frac{16 \pi p}{(n-1)\left(\alpha^{2}-n\right)} \frac{r_{+}^{2}}{\Xi}-\frac{4 q^{2}\left(\frac{r_{+}}{b}\right)^{2 \gamma(n-2)}}{\left(n-\alpha^{2}\right) r_{+}^{2(n-2)}} \\
\left.\times\left[\frac{1}{2(n-1)} \digamma_{1}\left(\eta_{+}\right)-\frac{1}{\left(\alpha^{2}+n-2\right)} \digamma_{2}\left(\eta_{+}\right)\right]\right\} \\
p=\left[\frac{(n-1)(n-2)}{16 \pi\left(\alpha^{2}-1\right) r_{+}^{2}}\left(\frac{r_{+}}{b}\right)^{2 \gamma}+\frac{(n-1) T}{4\left(1+\alpha^{2}\right) r_{+}}\right. \\
\left.+\frac{q^{2}}{8 \pi r_{+}^{2(n-1)}}\left(\frac{r_{+}}{b}\right)^{2 \gamma(n-2)} \digamma_{1}\left(\eta_{+}\right)\right] \Xi .
\end{gathered}
$$

Here, $r_{+}$is related to the thermodynamic volume $V$ as

$$
V=\frac{\varpi_{n-1}\left(1+\alpha^{2}\right)}{\left(n-\alpha^{2}\right)} r_{+}^{n} \begin{cases}\left(\frac{r_{+}}{b}\right)^{-\gamma(n-1)}, & \text { dilatonic BI, } \\ \left(\frac{r_{+}}{b}\right)^{-\frac{\gamma\left(n^{2}-4 n-1\right)}{n-3}}, & \text { BD-BI, }\end{cases}
$$

where

$$
\Xi= \begin{cases}1, & \text { dilatonic BI, } \\ \left(\frac{r_{+}}{b}\right)^{-\frac{4 \gamma}{n-3},}, & \text { BD-BI, }\end{cases}
$$

$$
\begin{aligned}
& \digamma_{1}(\eta)={ }_{2} F_{1}\left(\left[\frac{1}{2}, \frac{(n-3) \Upsilon}{\left(\alpha^{2}+n-2\right)}\right],\left[1+\frac{(n-3) \Upsilon}{\left(\alpha^{2}+n-2\right)}\right],-\eta\right), \\
& \digamma_{2}(\eta)={ }_{2} F_{1}\left(\left[\frac{1}{2}, \frac{(n-3) \Upsilon}{2(n-1)}\right],\left[1+\frac{(n-3) \Upsilon}{2(n-1)}\right],-\eta\right), \\
& \eta=\frac{q^{2}}{\beta^{2} r^{2(n-1)}}\left(\frac{r}{b}\right)^{2 \gamma(n-1)(n-5) /(n-3)}, \\
& \eta_{+}=\left.\eta(r)\right|_{r=r_{+}} \\
& \Upsilon=\frac{\alpha^{2}+n-2}{2 \alpha^{2}+n-3} .
\end{aligned}
$$

Now, to study the efficiency of the engine we cast our rectangular cycle in the Einstein frame as well as in the Jordan frame. Hereafter, for simplicity, we take $\beta \rightarrow \infty$.

The behavior of pressures in both frames can be seen from Fig. 9, which shows the rapid fall of pressures in Jordan frame. Moreover, the pressure of the isobar in the Einstein frame is higher than the pressure of the corresponding isobar in Jordan frame. In fact, the height of the cycle $\left(p_{1}-p_{3}\right)$ is higher for the Einstein frame which leads to more work. 


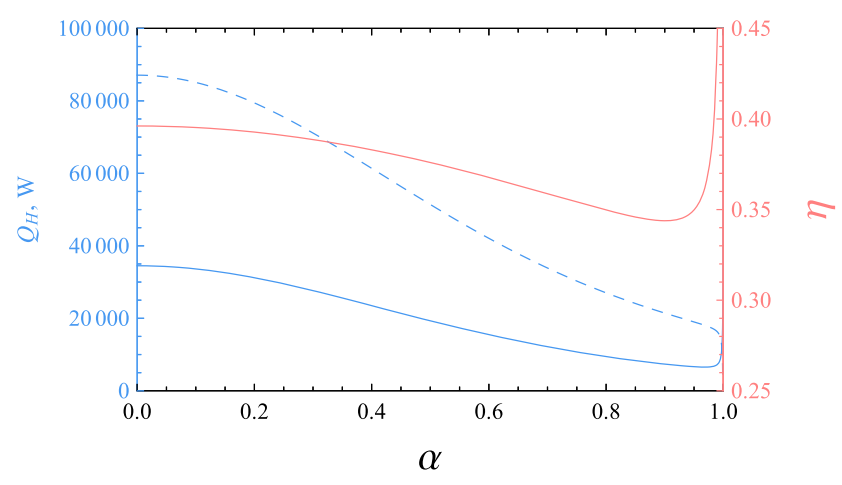

(a)

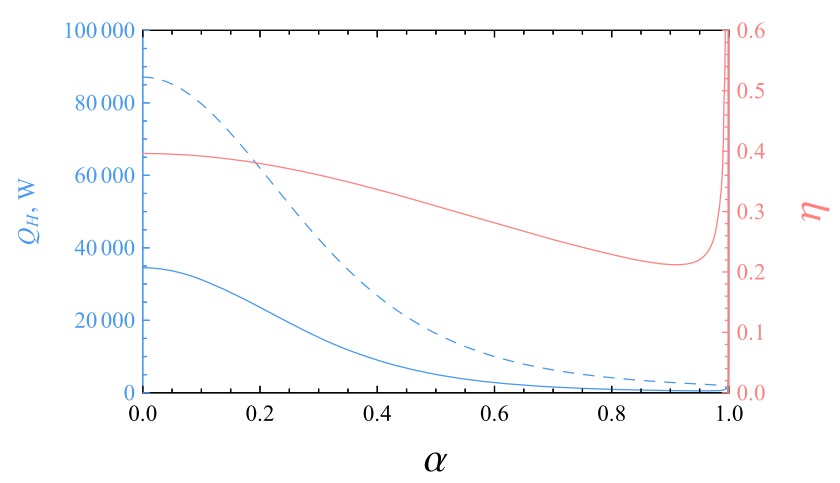

(b)

Fig. 10 Total work done (solid blue curve) and a net inflow of heat (dashed blue curve) for the engine are plotted on left $y$-axis, efficiency (solid red curve) is plotted on right $y$-axis for: a Einstein-BI-dilaton theory and $\mathbf{b}$ BD-BI theory (see the caption of Fig. 2 for parameter values)

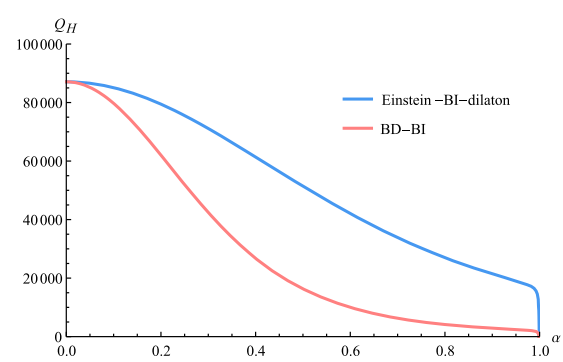

(a)

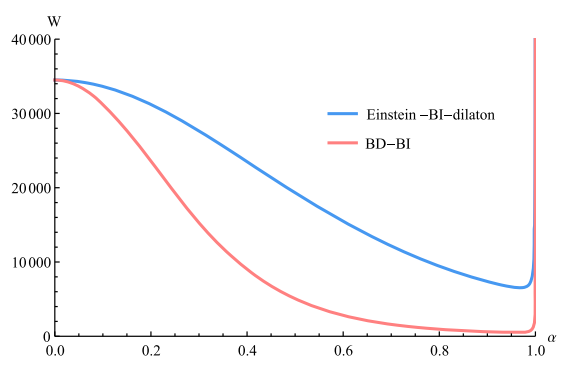

(b)

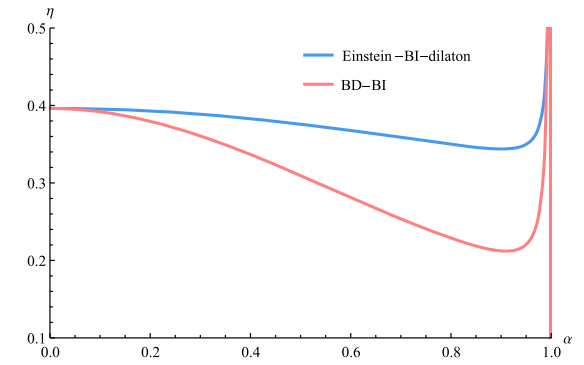

(c)

Fig. 11 Plots for (a) the net inflow of heat (b) total work done and (c) the efficiency vs. $\alpha$ (see the caption of Fig. 2 for parameter values)

From Fig. 10, we can see that as $\alpha$ increases, in Einstein frame, the inflow of heat monotonously decreases while the work done decreases up to $\alpha=0.97$ before raising. The efficiency decreases slowly up to $\alpha=0.9$ before a rapid rise. In the Jordan frame, $Q_{H}$ again decreases monotonously while $W$ decreases up to $\alpha=0.96$ then raises. The efficiency shows a similar behavior to the Einstein frame, however the minimum efficiency occurs at $\alpha=0.91$. Regardless of frames, maximum values of $Q_{H}$ and $W$ occur at $\alpha=0$, whereas efficiency reaches higher values when $\alpha \rightarrow 1$. Indeed, $Q_{H \text { Max }}=87089$ and $W_{\operatorname{Max}}=34499.7$.

For comparison, we plotted $Q_{H}, W$ and $\eta$ in Fig. 11. It can be seen that, for a given value of $\alpha$, engine running in Einstein frame takes more heat and generates more work and is also more efficient as compared to engine run in Jordan frame. This is so because the enthalpies (expressed in $r_{+}, p$ ) are not the same for each frame (although the expressions for the mass are the same). At a given value of $\alpha$, the enthalpy in BD theory dominates over the Einstein theory at the same pressure (Fig. 12), as well as at the same horizon radius $r_{+}$ (Fig. 12). This implies that at a given $\left(r_{+}, p\right)$, the enthalpy is higher in BD theory than in the Einstein theory.

Although BD theory dominates in enthalpy over Einstein theory, if we evaluate the enthalpies at the corners of the cycle, Einstein theory dominates over BD theory. This is because for a given volume, horizon radius $r_{+}$of the black hole is large in Einstein frame than that in the Jordan frame (see Fig. 13). Also, for a given $(V, T)$, the pressure is higher in the Einstein frame than that in the Jordan frame. Since the equations of state (expressed as $p(V, T)$ ) are not same for both frames (though Hawking temperatures have the same expressions).

In both frames, we find that the net inflow of heat $Q_{H}$, work $W$ and efficiency $\eta$ increase with $n$ (see Figs. 14, 15), while the allowed range of $\alpha$ decreases from the upper bound when regulated with Carnot efficiency $\eta_{C}$. In fact the window of the allowed values of $\alpha$ is wider in the Einstein frame than that in Jordan frame (see Table 1).

\section{Remarks}

We studied the effect of dilaton and Born-Infeld couplings on the efficiency of the holographic heat engines in Einstein gravity (with negative cosmological constant), where charged black hole is the working substance, in spite of the dependence of thermodynamic volume on dilaton coupling [68] and unusual asymptotics [87], $p d V$ terms exist [45] and mechanical work is extracted via the $p d V$ terms present in the first law of extended gravitational thermodynamics with a dynamical cosmological constant. In the case where the 


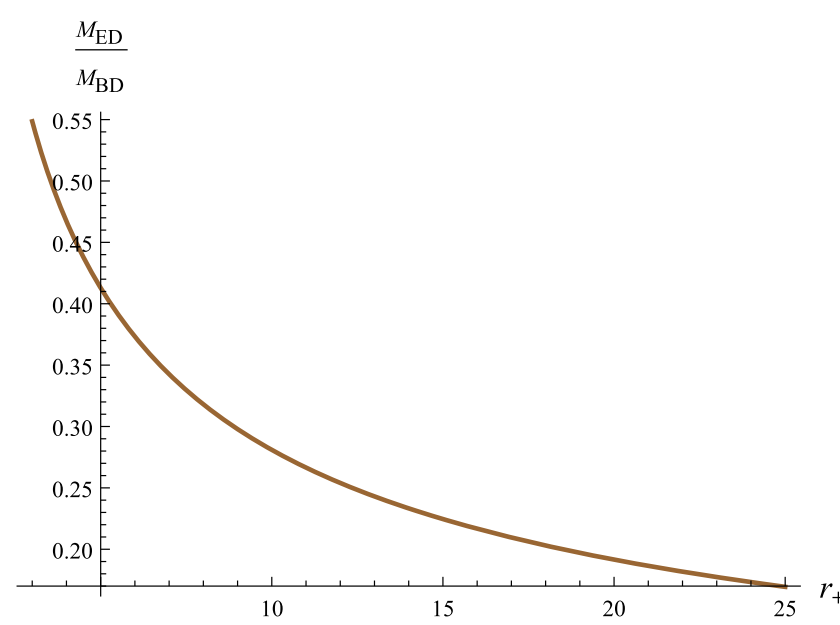

(a)

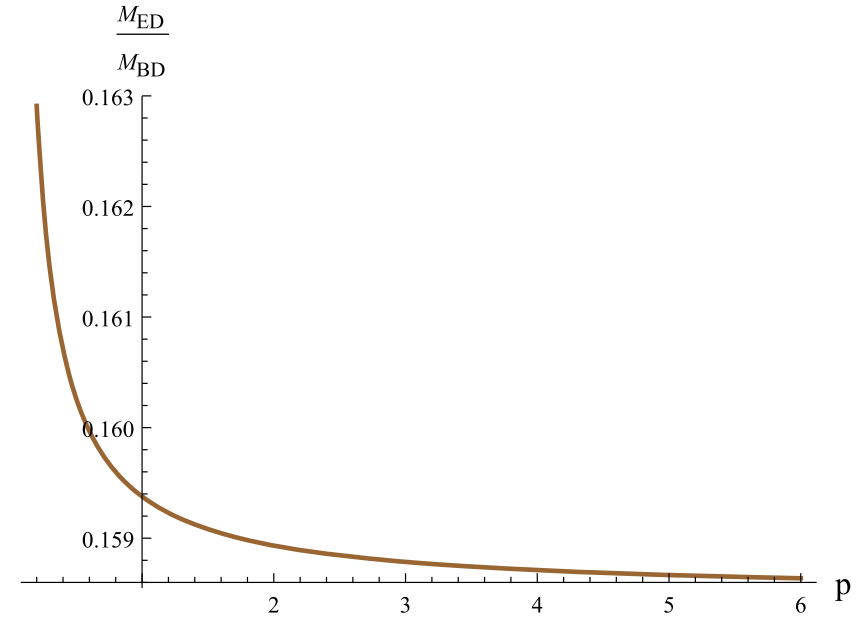

(b)

Fig. 12 Plots for the ratio of enthalpy in Einstein theory to enthalpy in BD theory $\left(M_{E D} / M_{B D}\right)$ a vs. $r_{+}$at $p=3, \alpha=0.4 \mathbf{b}$ vs. $p$ at $r_{+}=10, \alpha=0.5 .(n=4, b=1, q=0.1$.for both plots $)$

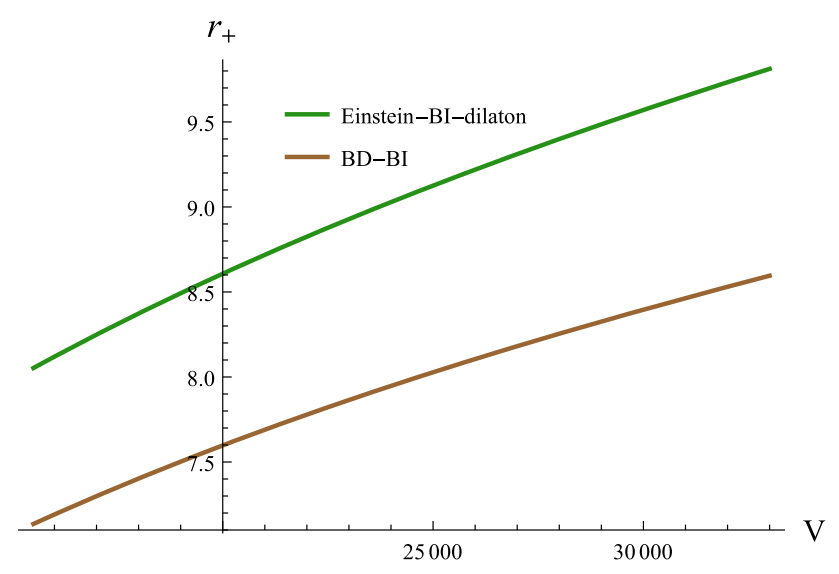

Fig. 13 Horizon radius $r_{+}$vs. volume $V$ for $\alpha=0.25, n=4, b=1$

dilaton coupling is absent, our exact result agrees with the high temperature calculation in [36]. As seen from Fig. 8, this behavior continues to hold even for non-zero values of dilaton coupling constant, signifying that the variations in the efficiency with $\beta$ are several orders of magnitude lower than that with $\alpha$. A similar feature was also noticed in the context of heat engines in Gauss-Bonnet black holes [36]. Increasing the parameter $q$ effects the efficiency significantly as seen from Fig. 3. We noticed in both Born-Infeld and dilaton cases that the increase in charge $q$ and volume $V_{2}$ lowers the efficiency, whereas the ratio $\frac{\eta}{\eta_{\mathrm{C}}}$ approaches unity on the account of increase in temperature $T_{2}$. Also for large $p_{1}$, leading behavior of the efficiency is $\left(1-\frac{p_{4}}{p_{1}}\right)$. In fact, increase in $q, V_{2}$ and $T_{2}$ implicitly changes the height of the cycle $\Delta p \equiv p_{1}-p_{4}$, which changes the efficiency accordingly [100].

We also compared the efficiency of engines in dilatonic Born-Infeld theory and Brans-Dicke-Born-Infeld theory. We see that our engine produces more work in the Einstein frame than in the Jordan frame. The Einstein frame provides a longer cycle along with high pressures for the corresponding isobars in the Jordan frame. Though black hole possesses more enthalpy in Brans-Dicke theory, for a fixed volume, the horizon radius is larger for an Einstein black hole. Hence, the

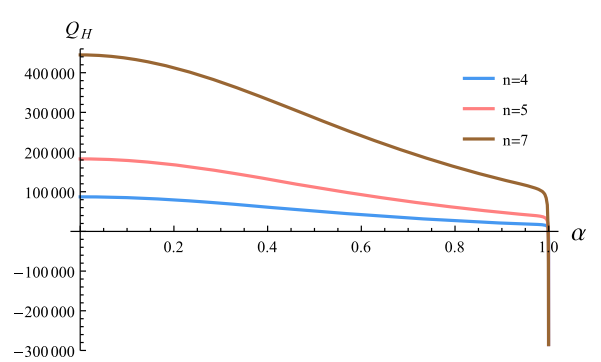

(a)

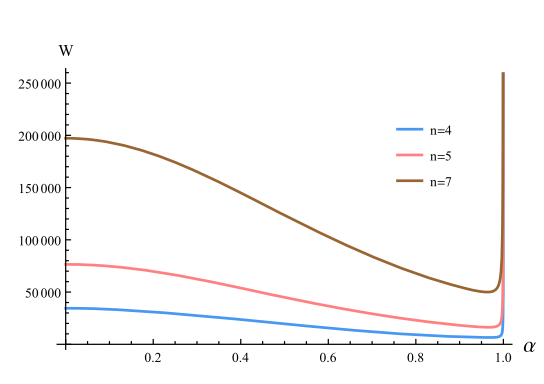

(b)

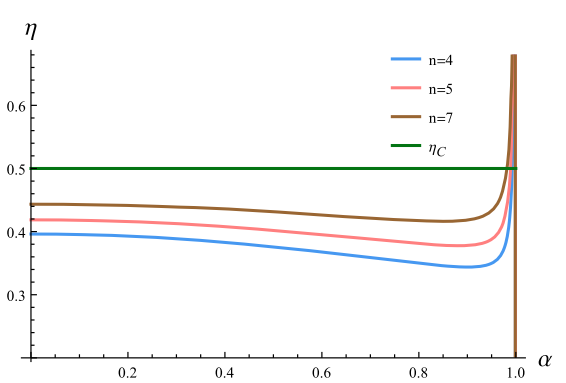

(c)

Fig. 14 In Einstein frame, plots for the net inflow of heat $Q_{H}$, work done $W$ and the efficiency $\eta$ with respect to $\alpha$ (see the caption of Fig. 2 for parameter values) 


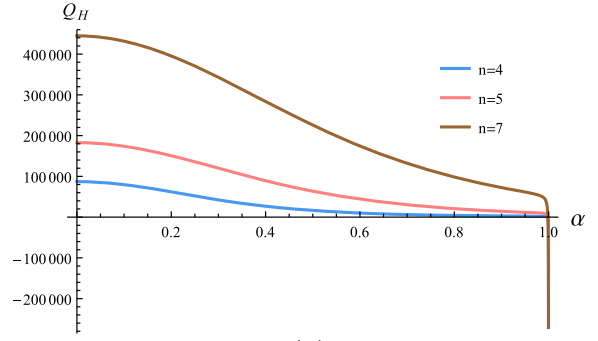

(a)

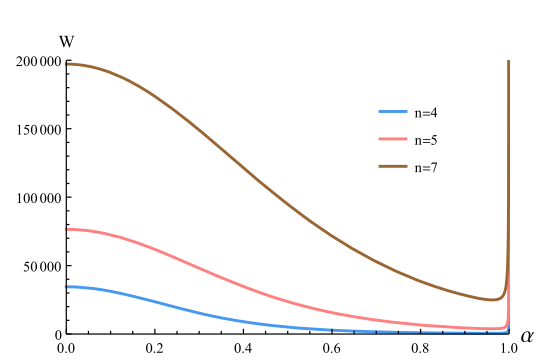

(b)

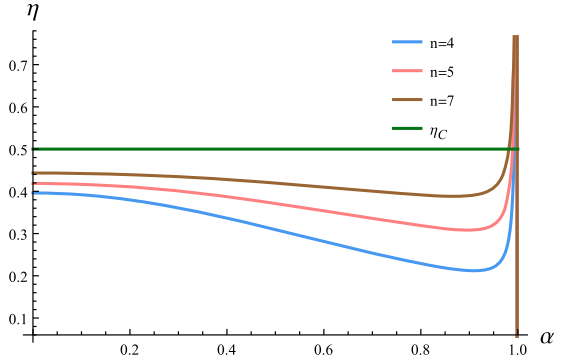

(c)

Fig. 15 In Jordan frame, plots for the net inflow of heat $Q_{H}$, work done $W$ and the efficiency $\eta$ with respect to $\alpha$ (see the caption of Fig. 2 for parameter values)

Table 1 Allowed range of $\alpha$ for the efficiency in Figs. 14 and 15

\begin{tabular}{lll}
\hline$n$ & BI-dilaton & BD-BI \\
\hline 4 & $0-0.992536$ & $0-0.992467$ \\
5 & $0-0.988880$ & $0-0.988811$ \\
7 & $0-0.981655$ & $0-0.981598$
\end{tabular}

calculation of $Q_{H}$ and efficiency $\eta$ as a function of enthalpies evaluated at the corners of the cycle yields larger values in the Einstein frame. We find that irrespective of the frames, the maximum values for $Q_{H}$ and $W$ occur at $\alpha=0$, while efficiency $\eta$ reaches to higher values when $\alpha \rightarrow 1$. We also checked that the qualitative behavior of the efficiency (as well as $Q_{H}, W$ ) does not alter in higher dimensions, although, the allowed range of $\alpha$ is small.

For both the dilatonic Born-Infeld and Brans-DickeBorn-Infeld cases, we choose a scheme where highest and lowest temperatures are held fixed to have a get close to the scheme independent answer. In this case, a comparison of our efficiency with two standards, i.e., the case $\eta_{0}$ (EinsteinMaxwell theory) and the Carnot efficiency $\eta_{C}$ could be performed. However, it would be nice to study the behavior of the efficiency in other possible schemes, as the equation of state still depends on coupling constants of the model and similar behavior is a priori not guaranteed. It would also be nice to have a better holographic understanding of heat engines which have been studied thus far, both with and without dilaton couplings and/or including other higher order gauge/gravity corrections to Einstein Gravity (whether asymptotically AdS/flat or not). An engine operating at the critical point could show further interesting scaling properties [101], especially in the large charge limit [102]. We leave these issues for future work.

Open Access This article is distributed under the terms of the Creative Commons Attribution 4.0 International License (http://creativecomm ons.org/licenses/by/4.0/), which permits unrestricted use, distribution, and reproduction in any medium, provided you give appropriate credit to the original author(s) and the source, provide a link to the Creative Commons license, and indicate if changes were made. Funded by SCOAP ${ }^{3}$.

\section{A Appendix}

The $(n+1)$-dimensional $(n \geq 3)$ action in which gravity is coupled to a dilaton and Born-Infeld fields is [23-26,44]

$$
\begin{aligned}
S= & \frac{1}{16 \pi} \int \mathrm{d}^{n+1} x \sqrt{-g}\left(\mathcal{R}-\frac{4}{n-1}(\nabla \Phi)^{2}-V(\Phi)\right. \\
& +L(F, \Phi)),
\end{aligned}
$$

where $\mathcal{R}$ is the Ricci scalar curvature, $\Phi$ is the dilaton field and $V(\Phi)$ is a potential for $\Phi$ :

$V(\Phi)=2 \Lambda_{0} e^{2 \zeta_{0} \Phi}+2 \Lambda e^{2 \zeta \Phi}$

where $\Lambda_{0}, \Lambda, \zeta_{0}$ and $\zeta$ are constants and the Born-Infeld $L(F, \Phi)$ part of the action is given by

$L(F, \Phi)=4 \beta^{2} e^{4 \alpha \Phi /(n-1)}\left(1-\sqrt{1+\frac{e^{-8 \alpha \Phi /(n-1)} F^{2}}{2 \beta^{2}}}\right)$.

Here, $\alpha$ is a constant determining the strength of the coupling of the scalar and electromagnetic fields, $F^{2}=F_{\mu \nu} F^{\mu \nu}$, where $F_{\mu \nu}=\partial_{\mu} A_{\nu}-\partial_{\nu} A_{\mu}$ is the electromagnetic field tensor, and $A_{\mu}$ is the electromagnetic vector potential. $\beta$ is the Born-Infeld parameter with the dimension of mass. A general solution is

$\mathrm{d} s^{2}=-f(r) \mathrm{d} t^{2}+\frac{\mathrm{d} r^{2}}{f(r)}+r^{2} R^{2}(r) h_{i j} \mathrm{~d} x^{i} \mathrm{~d} x^{j}$,

where $h_{i j}$ is a function of coordinates $x_{i}$ which spanned an $(n-1)$-dimensional hypersurface with constant scalar curvature $(n-1)(n-2) k$. Here $k$ is a constant characterizing the hypersurface (consider $k>0$ ). The electromagnetic field (finite at $r=0$ ) and charge are given, respectively, as 
$F_{t r}=\frac{\beta q e^{4 \alpha \Phi /(n-1)}}{\sqrt{\beta^{2}(r R)^{2 n-2}+q^{2}}}, \quad Q=\frac{q \omega_{n-1}}{4 \pi}$,

where $q$ is an integration constant related to the electric charge of the black hole and $\omega_{n-1}$ represents the volume of constant curvature hypersurface described by $h_{i j} d x^{i} d x^{j}$. $R(r)$ and $\Phi(r)$ are given, respectively, as

$R(r)=e^{2 \alpha \Phi /(n-1)}, \quad \Phi(r)=\frac{(n-1) \alpha}{2\left(1+\alpha^{2}\right)} \ln \left(\frac{b}{r}\right)$,

where $b$ is an arbitrary constant, $\gamma=\alpha^{2} /\left(\alpha^{2}+1\right)$.

The ADM (Arnowitt-Deser-Misner) mass $m$ of the black hole expressed in terms of the horizon radius $r_{h}$ is

$$
\begin{aligned}
m\left(r_{h}\right)= & -\frac{k(n-2)\left(\alpha^{2}+1\right)^{2} b^{-2 \gamma}}{\left(\alpha^{2}-1\right)\left(n+\alpha^{2}-2\right)} r_{h}{ }^{n-2+\gamma(3-n)} \\
& +\frac{2 \Lambda\left(\alpha^{2}+1\right)^{2} b^{2 \gamma}}{(n-1)\left(\alpha^{2}-n\right)} r_{h}^{n(1-\gamma)-\gamma} \\
& -\frac{4 \beta^{2}\left(\alpha^{2}+1\right)^{2} b^{2 \gamma}}{(n-1)\left(\alpha^{2}-n\right)} r_{h}^{n(1-\gamma)-\gamma} \\
& \times\left(1-2 F_{1}\left(\left[-\frac{1}{2}, \frac{\alpha^{2}-n}{2 n-2}\right],\right.\right. \\
& {\left.\left.\left[\frac{\alpha^{2}+n-2}{2 n-2}\right],-\eta\right)\right), }
\end{aligned}
$$

$$
\begin{aligned}
\zeta_{0} & =\frac{2}{\alpha(n-1)}, \quad \zeta=\frac{2 \alpha}{n-1}, \quad \Lambda_{0}=\frac{k(n-1)(n-2) \alpha^{2}}{2 b^{2}\left(\alpha^{2}-1\right)}, \\
\eta & =\frac{q^{2} b^{2 \gamma(1-n)}}{\beta^{2} r^{2(n-1)(1-\gamma)}} .
\end{aligned}
$$

Finally, $f(r)$ is given as

$$
\begin{aligned}
f(r)= & -\frac{k(n-2)\left(\alpha^{2}+1\right)^{2} b^{-2 \gamma}}{\left(\alpha^{2}-1\right)\left(n+\alpha^{2}-2\right)} r^{2 \gamma}-\frac{m}{r^{(n-1)(1-\gamma)-1}} \\
& +\frac{2 \Lambda\left(\alpha^{2}+1\right)^{2} b^{2 \gamma}}{(n-1)\left(\alpha^{2}-n\right)} r^{2(1-\gamma)} \\
& -\frac{4 \beta^{2}\left(\alpha^{2}+1\right)^{2} b^{2 \gamma} r^{2(1-\gamma)}}{(n-1)\left(\alpha^{2}-n\right)} \\
& \times\left(1-2 F_{1}\left(\left[-\frac{1}{2}, \frac{\alpha^{2}-n}{2 n-2}\right],\right.\right. \\
& {\left.\left.\left[\frac{\alpha^{2}+n-2}{2 n-2}\right],-\eta\right)\right) . }
\end{aligned}
$$

The temperature and entropy of the black hole are given, respectively, as

$$
\begin{aligned}
T_{+}= & -\frac{\left(\alpha^{2}+1\right) b^{2 \gamma} r_{+}^{1-2 \gamma}}{2 \pi(n-1)}\left(\frac{k(n-2)(n-1) b^{-4 \gamma}}{2\left(\alpha^{2}-1\right)} r_{+}^{4 \gamma-2}\right. \\
& \left.+\Lambda-2 \beta^{2}\left(1-\sqrt{1+\eta_{+}}\right)\right) \\
= & -\frac{k(n-2)\left(\alpha^{2}+1\right) b^{-2 \gamma}}{2 \pi\left(\alpha^{2}+n-2\right)} r_{+}^{2 \gamma-1} \\
& +\frac{\left(n-\alpha^{2}\right) m}{4 \pi\left(\alpha^{2}+1\right)} r_{+}^{(n-1)(\gamma-1)} \\
& -\frac{q^{2}\left(\alpha^{2}+1\right) b^{2(2-n) \gamma}}{\pi\left(\alpha^{2}+n-2\right)} r_{+}^{2(2-n)(1-\gamma)-1} \\
& \times{ }_{2} F_{1}\left(\left[\frac{1}{2}, \frac{n+\alpha^{2}-2}{2 n-2}\right],\left[\frac{3 n+\alpha^{2}-4}{2 n-2}\right],-\eta_{+}\right),
\end{aligned}
$$

$S=\frac{b^{(n-1) \gamma} \omega_{n-1} r_{+}^{(n-1)(1-\gamma)}}{4}$,

where $\eta_{+}=\eta\left(r=r_{+}\right)$. The gauge $A_{t}$ and electric potential $U$, measured at infinity with respect to the horizon are

$$
\begin{aligned}
A_{t}= & \frac{q b^{(3-n) \gamma}}{\Upsilon r^{\Upsilon}}{ }_{2} F_{1}\left(\left[\frac{1}{2}, \frac{\alpha^{2}+n-2}{2 n-2}\right],\right. \\
& {\left.\left[\frac{\alpha^{2}+3 n-4}{2 n-2}\right],-\eta\right), }
\end{aligned}
$$

where $\Upsilon=(n-3)(1-\gamma)+1$, and

$$
\begin{gathered}
U=\frac{q b^{(3-n) \gamma}}{\Upsilon r_{+}{ }^{\Upsilon}} F_{1}\left(\left[\frac{1}{2}, \frac{\alpha^{2}+n-2}{2 n-2}\right],\right. \\
\left.\left[\frac{\alpha^{2}+3 n-4}{2 n-2}\right],-\eta_{+}\right) .
\end{gathered}
$$

respectively.

\section{B Appendix}

Einstein-BI-dilaton gravity and its Brans-Dicke counterpart

The action of $(n+1)$ - dimensional BD theory, in which the dilaton field is decoupled from the matter field (electrodynamics) and coupled with gravity can be written as [45]

$$
\begin{aligned}
I_{B D-B I}= & -\frac{1}{16 \pi} \int_{\mathcal{M}} d^{n+1} x \sqrt{-g} \\
& \times\left(\Phi \mathcal{R}-\frac{\omega}{\Phi}(\nabla \Phi)^{2}-V(\Phi)+\mathcal{L}(\mathcal{F})\right),
\end{aligned}
$$

where $\mathcal{L}(\mathcal{F})$ is the Lagrangian of BI theory

$\mathcal{L}(\mathcal{F})=4 \beta^{2}\left(1-\sqrt{1+\frac{\mathcal{F}}{2 \beta^{2}}}\right)$, 
$\mathcal{R}$ is the Ricci scalar, $\omega$ is the coupling constant, $\Phi$ denotes the BD scalar field and $V(\Phi)$ is a self-interaction potential for $\Phi$.

Indeed, the BD-BI theory is conformally associated with the Einstein-BI-dilaton gravity. The appropriate conformal transformation is as follows:

$\bar{g}_{\mu \nu}=\Phi^{2 /(n-1)} g_{\mu \nu}$

where

$\bar{\Phi}=\frac{n-3}{4 \alpha} \ln \Phi$,

$\alpha=(n-3) / \sqrt{4(n-1) \omega+4 n}$.

By means of this conformal transformation, one finds that the action of BD-BI transforms to the well-known dilatonicBI gravity as

$$
\begin{aligned}
\bar{I}_{G}= & -\frac{1}{16 \pi} \int_{\mathcal{M}} d^{n+1} x \sqrt{-\bar{g}}\left\{\overline{\mathcal{R}}-\frac{4}{n-1}(\overline{\nabla \Phi})^{2}\right. \\
& -\bar{V}(\bar{\Phi})+\bar{L}(\overline{\mathcal{F}}, \bar{\Phi})\}
\end{aligned}
$$

where the potential $\bar{V}(\bar{\Phi})$ and the BI-dilaton coupling Lagrangian $\bar{L}(\bar{F}, \bar{\Phi})$ are, respectively,

$\bar{V}(\bar{\Phi})=\Phi^{-(n+1) /(n-1)} V(\Phi)$

and

$$
\begin{aligned}
\bar{L}(\overline{\mathcal{F}}, \bar{\Phi})= & 4 \beta^{2} e^{-4 \alpha(n+1) \bar{\Phi} /[(n-1)(n-3)]} \\
& \times\left(1-\sqrt{1+\frac{e^{16 \alpha \bar{\Phi} /[(n-1)(n-3)] \overline{\mathcal{F}}}}{2 \beta^{2}}}\right) .
\end{aligned}
$$

Black holes in Einstein frame (Einstein-dilaton-BI theory)

For the black hole solution, we assume the metric

$\mathrm{d} \bar{s}^{2}=-Z(r) \mathrm{d} t^{2}+\frac{\mathrm{d} r^{2}}{Z(r)}+r^{2} R^{2}(r) \mathrm{d} \Omega_{k}^{2}$,

and the potential $\overline{\mathbf{V}}(\bar{\Phi})$ as

$$
\begin{aligned}
\overline{\mathbf{V}}(\bar{\Phi})= & 2 \Lambda \exp \left(\frac{4 \alpha \bar{\Phi}}{n-1}\right)+\frac{k(n-1)(n-2) \alpha^{2}}{b^{2}\left(\alpha^{2}-1\right)} \\
& \times \exp \left(\frac{4 \bar{\Phi}}{(n-1) \alpha}\right)+\frac{W(r)}{\beta^{2}},
\end{aligned}
$$

where $\mathrm{d} \Omega_{k}^{2}$ betokens the Euclidean metric of an $(n-1)$ dimensional hypersurface with constant curvature $(n-1)(n-$ 2) $k$ and volume $\varpi_{n-1}$ (hereafter we take the option $k=1$ ).
Now, the metric (B.9) with the equations of motion for the action (B.6) admit the following solution:

$$
F_{t r}=E(r)=\frac{q e^{\left(\frac{4 \alpha \Phi(r)}{n-1}\right)}}{(r R(r))^{(n-1)} \sqrt{1+\frac{e^{\left(\frac{8 \alpha \Phi(r)}{n-3}\right)} q^{2}(r R(r))^{-2(n-1)}}{\beta^{2}}}},
$$$$
\bar{\Phi}=\frac{(n-1) \alpha}{2\left(1+\alpha^{2}\right)} \ln \left(\frac{b}{r}\right)
$$

$$
\begin{aligned}
& W(r)=\frac{4 q(n-1) \beta^{2} R(r)}{\left(1+\alpha^{2}\right) r^{\gamma} b^{n \gamma}} \int \frac{E(r)}{r^{n(1-\gamma)-\gamma}} \mathrm{d} r \\
& +\frac{4 \beta^{4}}{R(r)^{\frac{2(n+1)}{n-3}}}\left(1-\frac{E(r) R(r)^{(n-3)}}{q r^{1-n}}\right) \\
& -\frac{4 q \beta^{2} E(r)}{r^{n-1}}\left(\frac{r}{b}\right)^{\gamma(n-1)}, \\
& Z(r)=-\frac{k(n-2)\left(\alpha^{2}+1\right)^{2} b^{-2 \gamma} r^{2 \gamma}}{\left(\alpha^{2}+n-2\right)\left(\alpha^{2}-1\right)}+\left(\frac{\left(1+\alpha^{2}\right)^{2} r^{2}}{(n-1)}\right) \\
& \times \frac{2 \Lambda\left(\frac{r}{b}\right)^{-2 \gamma}}{\left(\alpha^{2}-n\right)}-\frac{m}{r^{(n-1)(1-\gamma)-1}} \\
& -\frac{4\left(1+\alpha^{2}\right)^{2} q^{2}\left(\frac{r}{b}\right)^{2 \gamma(n-2)}}{\left(n-\alpha^{2}\right) r^{2(n-2)}}\left(\frac{1}{2(n-1)} \digamma_{1}(\eta)\right. \\
& \left.-\frac{1}{\alpha^{2}+n-2} \digamma_{2}(\eta)\right) \text {, }
\end{aligned}
$$

where $m$ and $b$ are integration constants related to the mass and scalar field, respectively, and

$$
\begin{aligned}
& \digamma_{1}(\eta)={ }_{2} F_{1}\left(\left[\frac{1}{2}, \frac{(n-3) \Upsilon}{\alpha^{2}+n-2}\right],\left[1+\frac{(n-3) \Upsilon}{\alpha^{2}+n-2}\right],-\eta\right) \\
& \digamma_{2}(\eta)={ }_{2} F_{1}\left(\left[\frac{1}{2}, \frac{(n-3) \Upsilon}{2(n-1)}\right],\left[1+\frac{(n-3) \Upsilon}{2(n-1)}\right],-\eta\right) \\
& \Upsilon=\frac{\alpha^{2}+n-2}{2 \alpha^{2}+n-3} \\
& \eta=\frac{q^{2}\left(\frac{r}{b}\right)^{2 \gamma(n-1)(n-5) /(n-3)}}{\beta^{2} r^{2(n-1)}} \\
& R(r)=\exp \left(\frac{2 \alpha \Phi}{n-1}\right)=\left(\frac{r}{b}\right)^{-\gamma}
\end{aligned}
$$

\section{Black holes in Jordan frame (BD-BI theory)}

We invoke the conformal transformation to obtain black hole solutions of the BD-BI theory. The potential $\mathbf{V}(\Phi)$ in the Jordan frame using Eq. (B.7) is 


$$
\begin{aligned}
\mathbf{V}(\Phi)= & 2 \Lambda \Phi^{2}+\frac{k(n-1)(n-2) \alpha^{2}}{b^{2}\left(\alpha^{2}-1\right)} \\
& \Phi^{\left[(n+1)\left(1+\alpha^{2}\right)-4\right] /\left[(n-1) \alpha^{2}\right]}+\Phi^{(n+1) /(n-1)} \frac{W(r)}{\beta^{2}} .
\end{aligned}
$$

Taking into account the solutions in an Einstein frame with the mentioned conformal transformation, we are able to acquire the solutions of field equations for the BD-BI action (B.1). Considering the following $(n+1)$-dimensional metric:

$\mathrm{d} s^{2}=-A(r) \mathrm{d} t^{2}+\frac{\mathrm{d} r^{2}}{B(r)}+r^{2} H^{2}(r) \mathrm{d} \Omega_{k}^{2}$

we find that the functions $A(r)$ and $B(r)$ are

$$
\begin{aligned}
A(r) & =\left(\frac{r}{b}\right)^{4 \gamma /(n-3)} Z(r), \\
B(r) & =\left(\frac{r}{b}\right)^{-4 \gamma /(n-3)} Z(r), \\
H(r) & =\left(\frac{r}{b}\right)^{-\gamma\left(\frac{n-5}{n-3}\right)}, \\
\Phi(r) & =\left(\frac{r}{b}\right)^{-\frac{2 \gamma(n-1)}{n-3}} .
\end{aligned}
$$

\section{Thermodynamic quantities}

In both frames, the Hawking temperature, mass, entropy and the electric charge of the black hole are of the subsequent forms:

$$
\begin{aligned}
T= & \frac{\left(\alpha^{2}+1\right)}{2 \pi(n-1)}\left[-\frac{(n-2)(n-1)}{2\left(\alpha^{2}-1\right) r_{+}}\left(\frac{r_{+}}{b}\right)^{2 \gamma}\right. \\
& \left.-\Lambda r_{+}\left(\frac{r_{+}}{b}\right)^{-2 \gamma}+\Gamma_{+}\right], \\
M= & \frac{\varpi_{n-1} b^{(n-1) \gamma}}{16 \pi}\left(\frac{n-1}{1+\alpha^{2}}\right) m, \\
S= & \frac{\varpi_{n-1} b^{(n-1) \gamma}}{4} r_{+}^{(n-1)(1-\gamma)} . \\
Q= & \frac{q}{4 \pi},
\end{aligned}
$$

where

$$
\begin{aligned}
\Gamma_{+}= & -\frac{\left(\alpha^{2}+1\right)^{2} q^{2}}{2 \pi(n-1)}\left(\frac{r_{+}}{b}\right)^{2 \gamma(n-2)} r_{+}^{3-2 n} \digamma_{1}\left(\eta_{+}\right), \\
\eta_{+}=\left.\eta\right|_{r=r_{+}}, & \left(1+\alpha^{2}\right)^{2} r_{+}^{(n-1)(1-\gamma)-1}\left\{\frac{(n-2) b^{-2 \gamma}}{\left(1-\alpha^{2}\right)\left(n+\alpha^{2}-2\right)} r_{+}^{2 \gamma}\right. \\
m= & \frac{2 \Lambda r_{+}^{2}}{(n-1)\left(\alpha^{2}-n\right)}\left(\frac{r_{+}}{b}\right)^{-2 \gamma}
\end{aligned}
$$

$$
\begin{aligned}
& -\frac{4 q^{2}\left(\frac{r_{+}}{b}\right)^{2 \gamma(n-2)}}{\left(n-\alpha^{2}\right) r_{+}^{2(n-2)}} \times\left[\frac{1}{2(n-1)} \digamma_{1}\left(\eta_{+}\right)\right. \\
& \left.\left.-\frac{1}{\left(\alpha^{2}+n-2\right)} \digamma_{2}\left(\eta_{+}\right)\right]\right\} .
\end{aligned}
$$

In addition, in the extended phase space, thermodynamical pressure and volume are given by

$p=-\frac{\Lambda}{8 \pi} \times\left\{\begin{array}{cc}\left(\frac{r_{+}}{b}\right)^{-2 \gamma}, & \text { dilatonic BI } \\ \left(\frac{r_{+}}{b}\right)^{-\frac{2 \gamma(n-1)}{n-3}}, & \text { BD-BI }\end{array}\right.$

$V=\frac{\varpi_{n-1}\left(1+\alpha^{2}\right) r_{+}^{n}}{n-\alpha^{2}}\left\{\begin{array}{cc}\left(\frac{r_{+}}{b}\right)^{-\gamma(n-1)}, & \text { dilatonic BI } \\ \left(\frac{r_{+}}{b}\right)^{-\frac{\gamma\left(n^{2}-4 n-1\right)}{n-3}}, & \text { BD-BI. }\end{array}\right.$

\section{References}

1. M.M. Caldarelli, G. Cognola, D. Klemm, Thermodynamics of Kerr-Newman-AdS black holes and conformal field theories. Class Quant. Gravit. 17, 399-420 (2000). arXiv:hep-th/9908022 [hep-th]

2. S. Wang, S.-Q. Wu, F. Xie, L. Dan, The first laws of thermodynamics of the (2+1)-dimensional BTZ black holes and Kerrde Sitter spacetimes. Chin. Phys. Lett. 23, 1096-1098 (2006). arXiv:hep-th/0601147 [hep-th]

3. Y. Sekiwa, Thermodynamics of de Sitter black holes: thermal cosmological constant. Phys. Rev. D 73, 084009 (2006). arXiv:hep-th/0602269 [hep-th]

4. E.A. Larranaga Rubio, Stringy generalization of the first law of thermodynamics for rotating BTZ black hole with a cosmological constant as state parameter. arXiv:0711.0012 [gr-qc]

5. D. Kastor, S. Ray, J. Traschen, Enthalpy and the mechanics of AdS black holes. Class. Quant. Gravit. 26, 195011 (2009). arXiv:0904.2765 [hep-th]

6. B.P. Dolan, The cosmological constant and the black hole equation of state. Class. Quant. Gravit. 28, 125020 (2011). arXiv: 1008.5023 [gr-qc]

7. M. Cvetic, G. Gibbons, D. Kubiznak, C. Pope, Black hole enthalpy and an entropy inequality for the thermodynamic volume. Phys. Rev. D 84, 024037 (2011). arXiv:1012.2888 [hep-th]

8. B.P. Dolan, Compressibility of rotating black holes. Phys. Rev. D 84, 127503 (2011). arXiv:1109.0198 [gr-qc]

9. B.P. Dolan, Pressure and volume in the first law of black hole thermodynamics. Class. Quant. Gravit. 28, 235017 (2011). arXiv: 1106.6260 [gr-qc]

10. B.P. Dolan, Where is the PdV term in the fist law of black hole thermodynamics? arXiv:1209.1272 [gr-qc]

11. N. Altamirano, D. Kubiznak, R.B. Mann, Z. Sherkatghanad, Thermodynamics of rotating black holes and black rings: phase transitions and thermodynamic volume. Galaxies 2, 89-159 (2014). arXiv:1401.2586 [hep-th]

12. D. Kubiznak, R.B. Mann, M. Teo, Black hole chemistry: thermodynamics with lambda. Class. Quant. Gravit. 34, 063001 (2017). arXiv:1608.06147 [hep-th]

13. M. Henneaux, C. Teitelboim, The cosmological constant as a canonical variable. Phys. Lett. B 143, 415-420 (1984)

14. C. Teitelboim, The cosmological constant as a thermodynamic black hole parameter. Phys. Lett. B 158, 293-297 (1985) 
15. M. Henneaux, C. Teitelboim, The cosmological constant and general covariance. Phys. Lett. B 222, 195-199 (1989)

16. J.D. Bekenstein, Black holes and entropy. Phys. Rev. D 7, 23332346 (1973)

17. J.D. Bekenstein, Generalized second law of thermodynamics in black hole physics. Phys. Rev. D 9, 3292-3300 (1974)

18. S. Hawking, Particle creation by black holes. Commun. Math. Phys. 43, 199-220 (1975)

19. S. Hawking, Black holes and thermodynamics. Phys. Rev. D 13, 191-197 (1976)

20. M.K. Parikh, The volume of black holes. Phys. Rev. D 73, 124021 (2006). arXiv:hep-th/0508108 [hep-th]

21. C.V. Johnson, Thermodynamic volumes for AdS-Taub-NUT and AdS-Taub-Bolt. Class. Quant. Gravit. 31(23), 235003 (2014). arXiv: 1405.5941 [hep-th]

22. S.W. Hawking, C.J. Hunter, Gravitational entropy and global structure. Phys. Rev. D 59, 044025 (1999). arXiv:hep-th/9808085 [hep-th]

23. M.H. Dehghani, S. Kamrani, A. Sheykhi, P-V criticality of charged dilatonic black holes. Phys. Rev. D 90, 104020 (2014)

24. A. Sheykhi, Thermodynamical properties of topological BornInfeld-dilaton black holes. Int. J. Mod. Phys. D 18, 25 (2009)

25. A. Sheykhi, N. Riazi, Thermodynamics of black holes in $(n+1)$ dimensional Einstein-Born-Infeld dilaton gravity. Phys. Rev. D 75, 024021 (2007)

26. K.C.K. Chan, J.H. Horne, R.B. Mann, Charged dilaton black holes with unusual asymptotics. Nucl. Phys. B 447, 441 (1995)

27. A. Chamblin, R. Emparan, C.V. Johnson, R.C. Myers, Charged AdS black holes and catastrophic holography. Phys. Rev. D 60, 064018 (1999)

28. A. Chamblin, R. Emparan, C.V. Johnson, R.C. Myers, Holography, thermodynamics, and fluctuations of charged AdS black holes. Phys. Rev. D 60, 104026 (1999)

29. D. Kubiznak, R.B. Maan, P-V criticality of charged AdS black holes. JHEP 1207, 033 (2012). arxiv:1205.0559 [hep-th]

30. C.V. Johnson, Holographic heat engines. Class. Quant. Gravit. 31, 205002 (2014). arXiv:1404.5982 [hep-th]

31. A. Belhaj, M. Chabab, H. El Moumni, K. Masmar, M.B. Sedra, A. Segui, On heat properties of AdS black holes in higher dimensions. JHEP 05, 149 (2015). arXiv:1503.07308 [hep-th]

32. J. Sadeghi, K. Jafarzade, Heat engine of black holes. arXiv: 1504.07744 [hep-th]

33. E. Caceres, P.H. Nguyen, J.F. Pedraza, Holographic entanglement entropy and the extended phase structure of STU black holes. JHEP 09, 184 (2015). arXiv:1507.06069 [hep-th]

34. M.R. Setare, H. Adami, Polytropic black hole as a heat engine. Gen. Rel. Gravit. 47(11), 133 (2015)

35. C.V. Johnson, Gauss-Bonnet black holes and holographic heat engines beyond large N. Class. Quant. Gravit. 33(21), 215009 (2016). arXiv:1511.08782 [hep-th]

36. C.V. Johnson, Born-Infeld AdS black holes as heat engines. Class. Quant. Gravit. 33, 135001 (2016). arXiv:1512.01746 [hep-th]

37. C.V. Johnson, An exact efficiency formula for holographic heat engines. Entropy 18(4), 120 (2016). arXiv:1602.02838 [hep-th]

38. J.M. Maldacena, The large $\mathrm{N}$ limit of superconformal field theories and supergravity. Adv. Theor. Math. Phys. 2, 231-252 (1998). arXiv:hep-th/9711200 [hep-th]

39. E. Witten, Anti-de sitter space and holography. Adv. Theor. Math. Phys. 2, 253-291 (1998). arXiv:hep-th/9802150 [hep-th]

40. S.S. Gubser, I.R. Klebanov, A.M. Polyakov, Gauge theory correlators from non-critical string theory. Phys. Lett. B 428, 105-114 (1998). arXiv:hep-th/9802109 [hep-th]

41. E. Witten, Anti-de sitter space, thermal phase transition, and confinement in gauge theories. Adv. Theor. Math. Phys. 2, 505-532 (1998). arXiv:hep-th/9803131 [hep-th]
42. O. Aharony, S.S. Gubser, J.M. Maldacena, H. Ooguri, Y. Oz, Large $\mathrm{N}$ field theories, string theory and gravity. Phys. Rept. 323, 183386 (2000). arXiv:hep-th/9905111 [hep-th]

43. M.B. Green, J.H. Schwarz, E. Witten, Superstring theory (Cambridge University Press, Cambridge, 1987)

44. M.H. Dehghani, A. Sheykhi, Z. Dayyani, Critical behavior of Born-Infeld dilaton black holes. Phys. Rev. D 93(2), 024022 (2016). arXiv:1611.08978v1 [hep-th]

45. S.H. Hendi, R.M. Tad, Z. Armanfard et al, Extended phase space thermodynamics and $\mathrm{P}-\mathrm{V}$ criticality: Brans-Dicke-Born-Infeld vs. Einstein-Born-Infeld-dilaton black holes. Eur. Phys. J. C 76, 263 (2016). arXiv: 1511.02761 [gr-qc]

46. P.J. Steinhardt, F.S. Accetta, Hyperextended inflation. Phys. Rev. Lett. 64, 2740 (1990)

47. P.A.M. Dirac, A new basis for cosmology. Proc. R. Soc. Lond. A 165, 199 (1938)

48. S. Weinberg, Gravitation and cosmology (Wiley, Amsterdam, 1972)

49. A. Sheykhi, M.M. Yazdanpanah, Thermodynamics of charged Brans-Dicke AdS black holes. Phys. Lett. B 679, 311-316 (2009). arXiv:0904.1777 [hep-th]

50. S.H. Hendi, Z. Armanfard, Extended phase space thermodynamics and P-V criticality of charged black holes in Brans-Dicke theory. Gen. Relat. Gravity 47, 125 (2015). arXiv:1503.07070 [gr-qc]

51. M. Kord Zangeneh, M.H. Dehghani, A. Sheykhi, Thermodynamics of topological black holes in Brans-Dicke gravity with a power-law Maxwell field. Phys. Rev. D 92, 104035 (2015). arXiv: 1509.05990 [gr-qc]

52. S.H. Hendi, S. Panahiyan, B.E. Panah, et al, Phase transition of charged Black Holes in Brans-Dicke theory through geometrical thermodynamics. Eur. Phys. J. C 76, 396 (2016). arXiv: 1511.00598 [gr-qc]

53. A.D. Felice et al., Relaxing nucleosynthesis constraints on Brans-Dicke theories. Phys. Rev. D 74, 103005 (2006). arXiv:astro-ph/0510359

54. M. Born, L. Infeld, Foundations of the new field theory. Proc. R. Soc. A 144, 425 (1934)

55. R.G. Cai, D.W. Pang, A. Wang, Born-Infeld black holes in (A)dS spaces. arXiv:hep-th/0410158 [hep-th]

56. D. Kubiznak, R.B. Mann, P-V criticality of charged Ads black holes. J. High Energy Phys. 07, 033 (2012)

57. Sh Gunasekaran, D. Kubiznak, R.B. Mann, Extended phase space thermodynamics for charged and rotating black holes and BornInfeld vacuum polarization. J. High Energy Phys. 11, 110 (2012)

58. D.C. Zou, S.-J. Zhang, B. Wang, Critical behavior of Born-Infeld AdS black holes in the extended phase space thermodynamics. Phys. Rev. D 89, 044002 (2014)

59. R. Banerjee, D.R. Roychowdhury, Critical phenomena in BornInfeld AdS black holes. Phys. Rev. D 85, 044040 (2012)

60. R. Banerjee, D.R. Roychowdhury, Critical behavior of BornInfeld AdS black holes in higher dimensions. Phys. Rev. D 85, 104043 (2012)

61. J.X. Mo, W.B. Liu, P-V criticality of topological black holes in Lovelock-Born-Infeld gravity. Eur. Phys. J. C 74, 2836 (2014)

62. S.H. Hendi, S. Panahiyan, B. Panah, P-V criticality and geometrical thermodynamics of black holes with Born-Infeld type nonlinear electrodynamics. Int. J. Mod. Phys. D 25, 1650010 (2016)

63. S.H. Hendi, M.H. Vahidinia, Extended phase space thermodynamics and $\mathrm{P}-\mathrm{V}$ criticality of black holes with a nonlinear source. Phys. Rev. D 88, 084045 (2013)

64. D. Zou, Y. Liu, B. Wang, Critical behavior of charged GaussBonnet AdS black holes in the grand canonical ensemble. Phys. Rev. D 90, 044063 (2014) 
65. M.B. Jahani Poshteh, B. Mirza, Z. Sherkatghanad, Phase transition, critical behavior, and critical exponents of Myers-Perry black holes. Phys. Rev. D 88, 024005 (2013)

66. Z. Sherkatghanad, B. Mirza, Z. Mirzaeyan, S.A. Hosseini Mansoori, Critical behaviors and phase transitions of black holes in higher order gravities and extended phase spaces. Int. J. Mod. Phys. D 26, 1750017 (2017). arXiv:1412.5028 [gr-qc]

67. R. Zhao, H.H. Zhao, M.S. Ma, L.C. Zhang, On the critical phenomena and thermodynamics of charged topological dilaton AdS black holes. Eur. Phys. J. C 73, 2645 (2013). arXiv:1305.3725 [gr-qc]

68. A. Sheykhi, Thermodynamics of charged topological dilaton black holes. Phys. Rev. D 76, 124025 (2007). arXiv:0709.3619 [hep-th]

69. G.W. Gibbons, K. Maeda, Black holes and membranes in higherdimensional theories with dilaton fields. Phys. Lett. B 189, 29 (1987)

70. D. Brill, G. Horowitz, Negative energy in string theory. Phys. Lett. B 262, 437 (1991)

71. D. Garfinkle, G.T. Horowitz, A. Strominger, Charged black holes in string theory. Phys. Rev. D 43, 3140 (1991)

72. R. Gregory, J.A. Harvey, Black holes with a massive dilaton. Phys. Rev. D 47, 2411 (1993)

73. M. Rakhmanov, Dilaton black holes with electric charge. Phys. Rev. D 50, 5155 (1994)

74. L.J. Dixon, J.A. Harvey, String theories in ten-dimensions without space-time supersymmetry. Nucl. Phys. B 274, 93 (1986)

75. L. Alvarez-Gaume, P.H. Ginsparg, G.W. Moore, C. Vafa, An O(16) X O(16) heterotic string. Phys. Lett. B 171, 155 (1986)

76. A. Sagnotti, Some properties of open string theories. arXiv:hep-th/9509080 [hep-th]

77. A. Sagnotti, Surprises in open-string perturbation theory. Nucl. Phys. Proc. Suppl. 56B, 332-343 (1997). arXiv:hep-th/9702093 [hep-th]

78. S. Sugimoto, Anomaly cancellations in type I D9-D9-bar system and the USp(32) string. Progr. Theor. Phys. 102, 685-699 (1999). arXiv:hepth/9905159 [hep-th]

79. E. Dudas, J. Mourad, Brane solutions in strings with broken supersymmetry and dilaton tadpoles. Phys. Lett. B 486, 172-178 (2000). arXiv:hep-th/0004165 [hep-th]

80. C. Charmousis, D. Langlois, D. Steer, R. Zegers, Rotating spacetimes with a cosmological constant. JHEP 0702, 064 (2007). arXiv:gr-qc/0610091 [gr-qc]

81. G. Blaise, S. Jelena, et al, Holography for Einstein-Maxwelldilaton theories from generalized dimensional reduction. JHEP 1201, 089 (2012). arXiv:1110.2320v2 [hep-th]

82. O. Aharony, M. Berkooz, D. Kutasov, N. Seiberg, Linear dilatons, NS5-branes and holography. J. High Energy Phys. 10, 004 (1998). arXiv:hep-th/9808149 [hep-th]

83. S. Mignemi, D. Wiltshire, Spherically symmetric solutions in dimensionally reduced spacetimes. Class. Quant. Gravit. 6, 987 (1989)
84. D.L. Wiltshire, Spherically symmetric solutions in dimensionally reduced spacetimes with a higher-dimensional cosmological constant. Phys. Rev. D 44, 1100 (1991)

85. S. Mignemi, D.L. Wiltshire, Black holes in higher-derivative gravity theories. Phys. Rev. D 46, 1475 (1992)

86. S.J. Poletti, D.L. Wiltshire, Global properties of static spherically symmetric charged dilaton spacetimes with a Liouville potential. Phys. Rev. D 50, 7260 (1994). arXiv:gr-qc/9407021 [gr-qc]

87. K.C.K. Chan, J.H. Horne, R.B. Mann, Charged dilaton black holes with unusual asymptotics. Nucl. Phys. B 447, 441 (1995). arXiv:gr-qc/9502042v1 [gr-qc]

88. R.G. Cai, J.Y. Ji, K.S. Soh, Topological dilaton black holes. Phys. Rev. D 57, 6547 (1998)

89. R.G. Cai, Y.Z. Zhang, Holography and brane cosmology in domain wall backgrounds. Phys. Rev. D 64, 104015 (2001)

90. R.G. Cai, Y.Z. Zhang, Black plane solutions in four-dimensional spacetimes. Phys. Rev. D 54, 4891 (1996)

91. G. Clement, D. Gal'tsov, C. Leygnac, Linear dilaton black holes. Phys. Rev. D 67, 024012 (2003). arXiv:hep-th/0208225 [hep-th]

92. G. Clement, C. Leygnac, Non-asymptotically flat, non-AdS dilaton black holes. Phys. Rev. D 70, 084018 (2004). arXiv:gr-qc/0405034 [gr-qc]

93. T. Ghosh, P. Mitra, Asymptotically non-flat rotating dilaton black holes. Class. Quant. Gravit. 20, 1403 (2003). arXiv:gr-qc/0212057 [gr-qc]

94. A. Sheykhi, M.H. Dehghani, N. Riazi, J. Pakravan, Thermodynamics of rotating solutions in $(n+1)$-dimensional EinsteinMaxwell-dilaton gravity. Phys. Rev. D 74, 084016 (2006)

95. S.S. Yazadjiev, Einstein-Born-Infeld-dilaton black holes in nonasymptotically flat spacetimes. Phys. Rev. D 72, 044006 (2005). arXiv:hep-th/0504152 [hep-th]

96. M.H. Dehghani, S.H. Hendi, A. Sheykhi, H. Rastegar Sedehi, Thermodynamics of rotating black branes in Einstein-Born-Infeld-dilaton gravity.JCAP 0702 (2007) 020, arXiv:hep-th/0611288 [hep-th]

97. W.G. Brenna, Robert B. Mann, Miok Park, Mass and thermodynamic volume in Lifshitz spacetimes. Phys. Rev. D 92, 044015 (2015). arXiv:1505.06331 [hep-th]

98. Shamit Kachru, Xiao Liu, Michael Mulligan, Gravity duals of Lifshitz-like fixed points. Phys. Rev. D 78, 106005 (2008). arXiv:0808.1725 [hep-th]

99. J. Erdmenger, D. Fernandez, P. Goulart, P. Witkowski, Conductivities from attractors. JHEP 1703, 147 (2017). arXiv:1611.09381 [hep-th]

100. Ming Zhang, Wen-Biao Liu, $f(R)$ Black holes as heat engines. Int. J. Theor. Phys. 55, 5136 (2016)

101. C.V. Johnson, Approaching the Carnot limit at finite power: an exact solution. arXiv:1703.06119v1 [hep-th]

102. C.V. Johnson, Critical black holes in a large charge limit. arXiv:1705.01154 [hep-th] 